\title{
A STOCHASTIC MODEL FOR THE COOPERATIVE RELAXATION OF PROTEINS, BASED ON A HIERARCHY OF INTERACTIONS BETWEEN AMINO ACIDIC RESIDUES
}

\author{
M. ABUNDO*, L. ACCARDI and L. STELLA \\ Centro V. Volterra, Dipartimento di Matematica and \\ Dipartimento di Scienze e Tecnologie Chimiche, \\ Università Tor Vergata, 00193 Roma, Italy
}

N. ROSATO

Centro V. Volterra, Dipartimento di Medicina Sperimentale e Scienze Biochimiche, Università Tor Vergata, 00139 Roma, Italy

\author{
Communicated by P. Auger \\ Received 7 February 1996 \\ Revised 4 March 1997
}

\begin{abstract}
In this paper, a stochastic model for the cooperative relaxation of proteins, based on a hierarchic structure of the interactions between amino acids is proposed. It relies on the arbitrary splitting of interactions into two classes, strong and weak, and tests the preponderance of one class over the other. The presented model generalizes a first one valid for homogeneous interactions in the protein molecules previously studied by the authors. The time evolution of the system is studied as a function of five parameters, three of which are related to the cooperativity. Moreover, different approximations of the discrete system to a diffusion process, and to a Poisson process are considered, according to the magnitude of the parameters. A method for estimating the parameters from real data is proposed. Finally, numerical simulations and a comparison with the molecular dynamics of a real protein (Barnase) are reported.
\end{abstract}

\section{Introduction}

Proteins are essential elements of living organisms: they catalyze all the biochemical reactions that constitute life and perform several other functions. Their chemical structure is a chain of monomers (amino acids) covalently bonded. To be biologically active (e.g. as a catalyst) this chain must fold in a specific globular shape. The three-dimensional structure is determined by the sequence of different amino acids along the chain, but is realized through a complex network of non-covalent interactions (electrostatic, van der Waals, etc.) between monomers that are close in space, but that can be distant in the sequence.

${ }^{*}$ E-mail: abundo@mat.utovrm.it 
The problem of protein folding is extremely complex for two reasons: (a) the protein chain is highly flexible, and has an astronomically high number of possible conformations; (b) the non-covalent interactions are cooperative, in the sense that the energy of interaction between the monomers depends on all the other interactions.

For these reasons the prevision of the three-dimensional structure of a protein, starting from its amino acidic sequence, is at present impossible and is one of the most ambitious goals of contemporary molecular biophysics. Solving this puzzle would give us the ability to understand the functional mechanism of a newly discovered protein just by a simple chemical analysis and, more importantly, would make protein engineering easy and reliable, allowing the improvement of the activity and stability of proteins, the cure of genetic diseases, etc. just by changing a few amino acids along the sequence.

More generally, a change in the three-dimensional structure of a protein can happen for several reasons (binding of a ligand, change of external macroscopic parameters, etc.). The energy relaxation process that leads from the starting to the final equilibrium conformation is a phenomenon similar to protein folding, since it involves a rearrangement of the nonbonded interactions between amino acids.

In the previous papers, ${ }^{1,2}$ we have proposed a model of these processes of protein structure relaxation, based on the following simplifying assumptions:

(1) amino acids are considered as a whole unit, neglecting the details of their atomic composition;

(2) all amino acids are equivalent (their chemical structure is disregarded);

(3) every amino acid can interact with all the other monomers, regardless of their position along the chain;

(4) the interaction between two monomers is modelled as a two-state process. Two amino acids are either interacting or not; (therefore, we can speak of bonds even if we are treating non-covalent interactions);

(5) there is only one type of interaction;

(6) the formation or breaking of interactions between amino acids is a stochastic process;

(7) the probability of forming an additional interaction increases linearly with the number of interactions already formed (in this sense we speak of a cooperative phenomenon).

Assumption (3) derives from the flexibility of the protein chain and its ability to assume an enormous number of conformations. Assumption (6) is a result of the continuous thermal fluctuation of protein structure. Assumption (7) is a simple representation of the cooperativity described above. All the other hypotheses are just for the sake of simplicity.

The aim of such a crude model is to give a simple estimate of the degree of cooperativity involved in the folding of a specific protein, in order to compare different molecules and investigate possible correlations of their cooperativity with other characteristics (shape, flexibility, function, etc.). Applicability of the model 
needs the knowledge of the behavior, during relaxation, of an experimental or simulated parameter that can be taken as a measure of the degree of active interactions in the protein (e.g. the potential energy). The simplified time behavior of the model can be used to fit the data, obtaining in this way a value for the two parameters defined in Ref. 1. One of them can be assumed as a measure of the cooperativity of that specific protein. When this was done for a molecular dynamics simulation of the denaturation of a protein (BPTI), a significant value of cooperativity was detected. ${ }^{1}$

In this paper we try to improve our model by removing simplification (5). In fact, the various interactions that contribute to the folding of a protein have different physical causes, different potential energies and different radii of action.

For the sake of simplicity we consider here only two types of interactions, strong and weak, with different extents of potential energy difference between separate and coupled amino acids. We assume that the cooperative relaxation of proteins is based on a hierarchy of interactions between amino acids, with a predominant influence of the strong interactions on the weak ones. Once a strong interaction forms, several amino acids get closer and then can interact weakly more easily. If just a weak interaction is formed, non-appreciable difference for the formation of a strong one is produced. This is described by a generalization of the model proposed in Ref. 1, which introduces a hierarchy of interactions: the strong ones (denoted by "s") that can influence the weak ones (denoted by "w"), but not vice versa. In a practical example, we will try to identify these two types of interactions of the model with electrostatic and van der Waals forces, respectively. While the first one is long ranged (due to the $1 / r$ distance dependence of its potential), and can bring together amino acids that are far apart, the second one is weaker and short ranged (the attractive part of the potential has a $1 / r^{6}$ distance dependence) and has only a local influence.

The present model still gives a simple description of the cooperativity of the relaxation of a protein, with only five parameters. Two of them ( $p_{s}$ and $p_{w}$, see Sec. 2 for their definition) are related to the average probability of forming an interaction (strong and weak, respectively), which, in turn, is a function of the energy involved in the interaction. The other parameters $\left(\Delta p_{s}, \Delta p_{w}, \Delta p_{s w}\right.$, see Sec. 2 for definitions) express, respectively, the degree of cooperativity between the interactions of $s$-type, of $w$-type, and the influence of the number of $s$-type interactions on the formation of $w$-type ones.

In Sec. 2 we show how to describe the hierarchic model by a two-dimensional Markov chain (MC), in an analogous way as done in Ref. 1, where a one-dimensional MC was constructed for the model of cooperative interactions in proteins without hierarchic structure.

Section 3 is devoted to the study of the properties of the Markov chain. Moreover, generalizing the result obtained in Ref. 3, concerning the diffusion approximation of the one-dimensional MC for cooperative interactions without hierarchy, we obtain, in some range of values of the parameters, the continuous 
approximation of the two-dimensional MC to a diffusion process in $[0,1]^{2}$, as the number of allowed interactions goes to infinity. In another range of values of the parameters, we obtain an approximation of the MC to a Poisson process.

In the range of values of the parameters where the diffusion approximation holds, we get an estimate for the stationary probabilities and also information about the attainability of boundary states.

In Sec. 4 we show how the parameters of the model can be estimated from data representing the time dependence of the number of active strong and weak interactions, using the continuous approximation introduced in Sec. 3.

In Sec. 5 we report the results of the computer simulation and we discuss the applicability of our model to a real situation. We compare our model to the data relative to a molecular dynamics simulation of the thermal denaturation of the protein Barnase, appeared in Ref. 5 , and from these data we estimate the parameters of the model.

The results of this comparison are reported at the end of Sec. 5 and they show that our model, even with its crude simplifications, is satisfactorily applicable in the sense that it captures the qualitative behavior of cooperativity in the presence of a hierarchy of bonds.

\section{The Model}

Let there be a given number of particles (amino acidic residues) each of which can interact with one another by forming a bond. Suppose that two types of bonds are available: the strong ones $s$ and the weak ones $w$. Denote $N_{\text {tot }}$ the total number of permitted pairings among particles, we divide these pairings into two groups. The pairings linked by a bond of $s$-type belong to the first group; in the second group there are the pairings linked by $w$-type bonds. Let us suppose that the number of bonding of the first group is at most $N_{s}$, while that of the second group is at most $N_{w}$; then $N_{s}+N_{w}=N_{\text {tot }}$. The model described below is a generalization of the one studied in Ref. 1, where the interactions between residues were supposed to be all of the same type.

A configuration of the system is defined by a sequence of two-dimensional random variables (each component being a binary variable): $\left(\xi_{k}^{(j)}, \eta_{h}^{(j)}\right)_{k=1, \ldots, N_{s} ; h=1, \ldots, N_{w}}$ such that $\xi_{k}^{(j)}=+1$ if the $k$ th strong bond is activated at the discrete time $j$, $\xi_{k}^{(j)}=-1$, otherwise. Analogously, $\eta_{h}^{(j)}=+1$ if the $h$ th weak bond is activated at the discrete time $j, \eta_{h}^{(j)}=-1$, otherwise.

The evolution of the system, starting from an initial configuration $\left(\xi^{(0)}, \eta^{(0)}\right)$ is described in terms of the subtotal "energies" of the system at time $j$ :

$$
S_{N_{s}}\left(\xi^{(j)}\right)=\sum_{k=1}^{N_{s}} \xi_{k}^{(j)}, \quad S_{N_{w}}\left(\eta^{(j)}\right)=\sum_{h=1}^{N_{w}} \eta_{h}^{(j)}
$$

the total energy of the system at time $j$ being 


$$
S_{N}\left(\xi^{(j)}, \eta^{(j)}\right)=S_{N_{\bullet}}\left(\xi^{(j)}\right)+S_{N_{w}}\left(\eta^{(j)}\right) .
$$

Now, we introduce five parameters $p_{s}, p_{w}, \Delta p_{s}, \Delta p_{w}, \Delta p_{s w}$; our main assumption is that the r.v. $\xi_{1}^{(j)}, \ldots, \xi_{N_{s}}^{(j)}$ and $\eta_{1}^{(j)}, \ldots, \eta_{N_{w}}^{(j)}$ are independent and $\xi_{k}^{(j+1)}, k=$ $1, \ldots, N_{s}, \eta_{h}^{(j+1)}, h=1, \ldots, N_{w}$ are Bernoullian with distribution:

$$
\begin{gathered}
\operatorname{Pr}\left(\xi_{k}^{(j+1)}=+1 \mid S_{N}\left(\xi^{(j)}, \eta^{(j)}\right)\right)=p_{s}+\frac{\Delta p_{s}}{N_{s}} S_{N_{s}}\left(\xi^{(j)}\right) \\
\operatorname{Pr}\left(\eta_{h}^{(j+1)}=+1 \mid S_{N}\left(\xi^{(j)}, \eta^{(j)}\right)\right)=\left(p_{w}+\Delta p_{s w}\right)+\frac{\Delta p_{w}}{N_{w}} S_{N_{w}}\left(\eta^{(j)}\right)+\frac{\Delta p_{s w}}{N_{s}} S_{N_{s}}\left(\xi^{(j)}\right)
\end{gathered}
$$

The equality (2.2) implies that the probability to form a bond of $s$-type at time $j+1$, depends only on the relative number of bonds of $s$-type already present at time $j$, through the coefficients $p_{s}, \Delta p_{s}$. Then, if $\Delta p_{s}$ is large we have a great probability to create bonds of $s$-type at the new instant of time. Moreover, for fixed $p_{s}$ and $\Delta p_{s}$, the greater the energy $S_{N_{s}}$, at time $j$, the greater the probability that bonds of $s$-type may be formed at time $j+1$. On the contrary, (2.3) means that the probability of forming a bond of $w$-type at time $j+1$, depends both on the relative number of bonds of $w$-type and the relative number of bonds of stype, already present at time $j$, through the coefficients $p_{w}, \Delta p_{w}, \Delta p_{s w}$. Moreover, for a fixed situation of the $w$-type bonds, the greater the energy $S_{N_{s}}$ of $s$-type bonds at time $j$, the greater the probability that bonds of $w$-type may be formed at time $j+1$, i.e. the presence of strong bonds among particles makes the formation of weak ones easier.

The parameters $p_{s}$ and $p_{w}$ may be called the mean, or ground probabilities for bonds of $s$-type and $w$-type, respectively; in fact, they represent the probability of forming a bond of $s$-type (respectively $w$-type) at zero energy of the strong bonds (resp. at zero energy of the weak bonds and with $\Delta p_{s w}=0$ ). Physically, $p_{s}$ and $p_{w}$ are decreasing functions of the bonding energy for the formation of a bond of $s$-type and $w$-type, respectively.

We call $\Delta p_{s}$ and $\Delta p_{w}$ the s-coupling capacity and w-coupling capacity, respectively. $\Delta p_{s}$ represents the maximum allowed increment of the probability to form a bond of $s$-type for given $p_{s} ; \Delta p_{w}$ represents the maximum allowed increment of the probability to form a bond of $w$-type, for given $p_{w}$, under the condition $S_{N_{s}}=0$, and $\Delta p_{s w}=0$. Finally, $\Delta p_{s w}$ represents the cross-coupling capacity. When $\Delta p_{s w}=0$, the processes $\left\{\xi_{k}^{(j)}\right\}$ and $\left\{\eta_{k}^{(j)}\right\}$ are independent and the number of stype bonds does not influence the formation of $w$-type bonds. When $\Delta p_{s w} \neq 0$, the two processes are correlated and an increase of $\Delta p_{s w}$ results in a more effective influence of the number of $s$-type bonds on the formation of $w$-type bonds.

Modeling the protein system in this way renders well enough the picture of auto and cross-cooperativity among different types of interactions (bonds) between amino acidic residues. In the following, in order to make (2.2), (2.3) well-defined we shall impose the following consistency conditions: 


$$
\left\{\begin{array}{l}
p_{s} \geq \Delta p_{s} \geq 0, \quad p_{s}+\Delta p_{s} \leq 1 \\
p_{w} \geq \Delta p_{w} \geq 0, \quad p_{w}+\Delta p_{w} \leq 1 \\
0 \leq p_{w}+\Delta p_{w}+2 \Delta p_{s w} \leq 1, \quad-\frac{1}{2} \leq \Delta p_{s w} \leq \frac{1}{2}
\end{array}\right.
$$

Proceeding in analogy to Ref. 1 , by a suitable change of variables, we look at the twodimensional process $\left(X_{n}, Y_{n}\right), n=1,2, \ldots$ whose components represent the actual number of bonds of $s$-type and $w$-type (at discrete time $n$ ). Conditions (2.2), (2.3) imply that the events $\left\{X_{n+1}=x\right\}$ and $\left\{Y_{n+1}=y\right\}$ are conditionally independent given the event $\left\{X_{n}=x^{\prime}, Y_{n}=y^{\prime}\right\}$, i.e.:

$$
\begin{aligned}
& \operatorname{Pr}\left(X_{n+1}=x, Y_{n+1}=y \mid X_{n}=x^{\prime}, Y_{n}=y^{\prime}\right) \\
& \quad=\operatorname{Pr}\left(X_{n+1}=x \mid X_{n}=x^{\prime}, Y_{n}=y^{\prime}\right) \cdot \operatorname{Pr}\left(Y_{n+1}=y \mid X_{n}=x^{\prime}, Y_{n}=y^{\prime}\right) .
\end{aligned}
$$

Moreover, we suppose that:

$$
\operatorname{Pr}\left(X_{n+1}=x \mid X_{n}=x^{\prime}, Y_{n}=y^{\prime}\right)=\operatorname{Pr}\left(X_{n+1}=x \mid X_{n}=x^{\prime}\right),
$$

i.e. the event $\left\{X_{n+1}=x^{\prime}\right\}$ is independent of $\left\{Y_{n}=y^{\prime}\right\}$. All that leads to the construction of a two-dimensional Markov chain $\left(X_{n}, Y_{n}\right)$ with state space $\Omega=$ $\left\{\left(i_{s}, i_{w}\right): i_{s} \in\left\{0,1, \ldots, N_{s}\right\}, i_{w} \in\left\{0,1, \ldots, N_{w}\right\}\right\}$ and transition probabilities given by $^{1}$ :

$$
\begin{aligned}
P_{\left(i_{s}, i_{w}\right)\left(j_{s}, j_{w}\right)}= & \operatorname{Pr}\left(\left(X_{n+1}=j_{s}, Y_{n+1}=j_{w}\right) \mid\left(X_{n}=i_{s}, Y_{n}=j_{w}\right)\right) \\
= & \left(\begin{array}{c}
N_{s} \\
j_{s}
\end{array}\right)\left(p_{s}-\Delta p_{s}+2 \Delta p_{s} \frac{i_{s}}{N_{s}}\right)^{j_{s}}\left(1-p_{s}+\Delta p_{s}-2 \Delta p_{s} \frac{i_{s}}{N_{s}}\right)^{N_{s}-j_{s}} \\
& \times\left(\begin{array}{c}
N_{w} \\
j_{w}
\end{array}\right)\left(p_{w}-\Delta p_{w}+2 \Delta p_{w} \frac{i_{w}}{N_{w}}+2 \Delta p_{s w} \frac{i_{s}}{N_{s}}\right)^{j_{w}} \\
& \times\left(1-p_{w}+\Delta p_{w}-2 \Delta p_{w} \frac{i_{w}}{N_{w}}-2 \Delta p_{s w} \frac{i_{s}}{N_{s}}\right)^{N_{w}-j_{w}}
\end{aligned}
$$

It is easy to verify that $\sum_{\left(j_{s}, j_{w}\right)} P_{\left(i_{s}, i_{w}\right)\left(j_{s}, j_{w}\right)}=1$, so that the quantities in (2.7) are indeed transition probabilities.

\section{The Properties of the Markov Chain}

\subsection{Absorption problems}

(i) The case when $p_{s}=\Delta p_{s} \neq 1 / 2$ and $p_{w}=\Delta p_{w} \neq 1 / 2$ :

From (2.7) we obtain that $P_{(0,0)(0,0)}=1$ and $P_{(0,0)\left(j_{s}, j_{w}\right)}=0, \forall\left(j_{s}, j_{w}\right) \neq$ $(0,0)$. Therefore the state $(0,0)$ is absorbing. In terms of the variable $\xi_{i}, \eta_{i}$, the state $(0,0)$ corresponds to the subtotal energies $S_{N_{s}}(\xi)=-N_{s}, S_{N_{w}}(\eta)=$ $-N_{w}$, that is to the case of full uncoupling for both types of bonds. 
(ii) The case when $p_{s}=\Delta p_{s} \neq 1 / 2$ and $p_{w}+\Delta p_{w}=1$ :

From (2.7), we obtain that $P_{\left(0, N_{w}\right)\left(0, N_{w}\right)}=1$ and $P_{\left(0, N_{w}\right)\left(j_{s}, j_{w}\right)}=0 \forall\left(j_{s}, j_{w}\right) \neq$ $\left(0, N_{w}\right)$. The state $\left(0, N_{w}\right)$ is absorbing; it corresponds to a situation of full coupling for $w$-type bonds and full uncoupling for $s$-type bonds.

(iii) The case when $p_{s}+\Delta p_{s}=1$ and $\Delta p_{w}=p_{w}+2 \Delta p_{s w}$ :

This is compatible with conditions (2.4) only if $\Delta p_{s w} \leq 0$. From (2.7), we obtain that $P_{\left(N_{s}, 0\right)\left(N_{s}, 0\right)}=1$ and $P_{\left(N_{s}, 0\right)\left(j_{s}, j_{w}\right)}=0 \forall\left(j_{s}, j_{w}\right) \neq\left(N_{s}, 0\right)$. The state $\left(N_{s}, 0\right)$ is absorbing; it corresponds to a situation of full coupling for bonds of $s$-type and full uncoupling for $w$-type bonds. Thus, the bonds of $s$-type do not cooperate to increase the number of bonds of $w$-type, but vice versa. In fact, the cross-coupling capacity is negative. The case (iii) is perhaps only a mathematical feature of the model.

(iv) The case when $p_{s}+\Delta p_{s}=1$ and $p_{w}+\Delta p_{w}+2 \Delta p_{s w}=1$ :

From (2.7), we obtain that $P_{\left(N_{s}, N_{w}\right)\left(N_{s}, N_{w}\right)}=1$ and $P_{\left(N_{s}, N_{w}\right)\left(j_{s}, j_{w}\right)}=0$ $\forall\left(j_{s}, j_{w}\right) \neq\left(N_{s}, N_{w}\right)$. The state $\left(N_{s}, N_{w}\right)$ is absorbing; it corresponds to a situation of full coupling for both types of bonds.

(v) The case when $p_{s}>\Delta p_{s}>0, p_{s}+\Delta p_{s}<1, p_{w}>\Delta p_{w}>0, p_{w}+\Delta p_{w}<1$, $p_{w}+\Delta p_{w}+2 \Delta p_{s w}<1:$

It is the most interesting case from the physical point of view. As easily seen, $P_{\left(i_{s}, i_{w}\right)\left(j_{s}, j_{w}\right)}>0 \forall\left(i_{s}, i_{w}\right),\left(j_{s}, j_{w}\right)$ which implies that the chain is irreducible. Indeed, the second and fourth factors in (2.7) are evidently positive, as well the third and the fifth ones, because

(a) $1-p_{s}+\Delta p_{s}-2 \Delta p_{s} \frac{i_{s}}{N_{s}} \geq 1-p_{s}+\Delta p_{s}-2 \Delta p_{s}=1-p_{s}-\Delta p_{s}>0$,

(b) $1-p_{w}+\Delta p_{w}-2 \Delta p_{w} \frac{i_{w}}{N_{w}}-2 \Delta p_{s w} \frac{i_{s}}{N_{s}} \geq 1-p_{w}+\Delta p_{w}-2 \Delta p_{w}-2 \Delta p_{s w}$

$$
=1-p_{w}-\Delta p_{w}-2 \Delta p_{s w}>0 \text {. }
$$

\subsection{The stationary probabilities}

In the case $(v)$ above, since the $\mathrm{MC}$ is irreducible, the stationary probabilities $\Pi_{\mathbf{i}}=\Pi_{\left(i_{s}, i_{w}\right)}, i_{s}=0,1, \ldots, N_{s}, \mathbf{1}_{w}=0,1, \ldots, N_{w}$ exist such that $\Pi_{\mathbf{i}}=\lim _{n \rightarrow \infty} P_{\mathbf{k i}}^{(n)}$, irrespective of the initial state $\mathbf{k}=\left(k_{s}, k_{w}\right)$, where $P_{\mathbf{k i}}^{(n)}$ is the probability of transition from the state $\mathbf{k}=\left(k_{s}, k_{w}\right)$ into the state $\mathbf{i}=\left(i_{s}, i_{w}\right)$ in $n$ steps. Moreover, $\lim _{n \rightarrow \infty} \operatorname{Pr}\left(\left(X_{n}, Y_{n}\right)=\mathbf{i}\right)=\Pi_{\mathbf{i}}$.

The stationary probability $\Pi_{i}$ represents the probability that the system is in the state $i$ after an infinite time, irrespective of the initial state. Thus, the knowledge of the stationary probabilities gives information about the fluctuations of the system at the equilibrium.

The stationary probabilities $\Pi_{\mathbf{i}}=\Pi_{\left(i_{s}, i_{w}\right)}$ can be computed by solving the equation:

$$
\Pi_{\mathrm{i}}=\sum_{\mathrm{j}} \Pi_{\mathrm{j}} P_{\mathrm{ji}} .
$$


This equation is nothing but the equation which yields the left eigenvector of the matrix $\mathbf{P}$ with eigenvalue 1. In practice, since the index $\mathbf{i}$ is two-dimensional, when $N_{s}, N_{w}$ are large, Eq. (3.1) becomes computationally intractable. The stationary probabilities $\Pi_{\mathbf{i}}$ and the marginal stationary probabilities $\pi_{i_{s}}=\sum_{i_{w}=0}^{N_{w}} \Pi_{\left(i_{s}, i_{w}\right)}$ and $\pi_{i_{w}}=\sum_{i_{s}=0}^{N_{s}} \Pi_{\left(i_{s}, i_{w}\right)}$ can be numerically found by computing the frequencies of visits to each state $i_{s} \in\left\{0,1, \ldots, N_{s}\right\}, j_{s} \in\left\{0,1, \ldots, N_{w}\right\}$ following a long enough trajectory of the system.

In Sec. 5, we shall present some numerical simulations and estimations of stationary probabilities. Since Eq. (3.1) is theoretically intractable, to find an analytical estimate of stationary probabilities for $N_{s}, N_{w}$ large, we can proceed as was done in Ref. 1 for the analogous one-dimensional Markov chain. Indeed, a heuristic application of the law of large numbers (cf. also Ref. 8) suggests that the following limits hold in probability:

$$
\begin{gathered}
\frac{1}{N_{s}} \cdot \lim _{j \rightarrow \infty} X_{j} \rightarrow^{P} a_{s}=\frac{p_{s}-\Delta p_{s}}{1-2 \Delta p_{s}} \text { as } N_{s} \rightarrow \infty \\
\frac{1}{N_{w}} \cdot \lim _{j \rightarrow \infty} Y_{j} \rightarrow^{P} a_{w}=\frac{\left(1-2 \Delta p_{s}\right)\left(p_{w}-\Delta p_{w}\right)+2 \Delta p_{s w}\left(p_{s}-\Delta p_{s}\right)}{\left(1-2 \Delta p_{s}\right)\left(1-2 \Delta p_{w}\right)}, \text { as } N_{w} \rightarrow \infty
\end{gathered}
$$

Then, we obtain the following approximation in probability of the stationary probabilities, for $N_{s}, N_{w}$ large:

$$
\begin{aligned}
\Pi_{\left(n_{s}, n_{w}\right)} & =\lim _{k \rightarrow \infty} \operatorname{Pr}\left(X_{k}=n_{s}, Y_{k}=n_{w}\right) \\
& \sim\left(\begin{array}{l}
N_{s} \\
n_{s}
\end{array}\right) a_{s}^{n_{s}}\left(1-a_{s}\right)^{N_{s}-n_{s}}\left(\begin{array}{c}
N_{w} \\
n_{w}
\end{array}\right) a_{w}^{n_{w}}\left(1-a_{w}\right)^{N_{w}-n_{w}}
\end{aligned}
$$

However, this is a rather rough estimate, since in the limit $N_{s}, N_{w} \rightarrow \infty$, the processes $X_{k}, Y_{k}$ appear to be independent and binomial distributed with parameters $a_{s}$ and $a_{w}$, while they are known to be correlated.

\subsection{The diffusion approximation (for $p_{i}, \Delta p_{i}$ close to $1 / 2$ and $\Delta p_{s w}$ close to zero)}

Another way to obtain information about the stationary probabilities is to consider the continuous approximation of the two-dimensional MC with transition probabilities matrix (2.7), to a diffusion process in $[0,1]^{2}$, in analogous way, as was done in Ref. 3, where in the case of protein interaction without hierarchic structure, we have found a continuous approximation of the $\mathrm{MC}$ to a diffusion process in $[0,1]$. In fact, once the stationary distribution of the diffusion process is known, by discretization, the stationary probabilities can be approximately obtained.

We observe that the diffusion approximation also represents a convenient tool for fitting experimental or simulated data with our model; indeed this approach will be followed in Secs. 4 and 5 in estimating the parameters. 
Now we state the diffusion approximation in the case of a hierarchic structure between bonds.

Let us introduce some notations. Set $N_{1}=N_{s}=N, N_{2}=N_{w}, \mathbf{N}=\left(N_{1}, N_{2}\right), i_{1}=$ $i_{s}, i_{2}=i_{w}$, and $K_{N}=K_{N_{1}, N_{2}}=\left\{\left(u_{1}, u_{2}\right)=\left(i_{1} / N_{1}, i_{2} / N_{2}\right), i_{1} \in\left\{0,1, \ldots, N_{1}\right\}, i_{2} \in\right.$ $\left.\left\{0,1, \ldots, N_{2}\right\}\right\}$ and let

$$
\chi^{\mathbf{N}}(t)=\left(\chi_{1}^{\mathbf{N}}(t), \chi_{2}^{\mathbf{N}}(t)\right)=\left(\frac{X^{N_{1}}}{N_{1}}([N t]), \frac{Y^{N_{2}}}{N_{2}}([N t])\right)
$$

be the two-dimensional rescaled process with values in $K_{\mathrm{N}}$, where $[z]$ denotes the integer part of $z$, i.e. the largest integer $\leq z$.

Here, $X^{N_{1}}(k), Y^{N_{2}}\left(k^{\prime}\right)$ are indeed the same processes considered in (2.5), (2.7) where, for simplicity, the superscripts $N_{1}, N_{2}$ are omitted.

We are interested in the behavior of the process $\chi^{\mathbf{N}}(t)$ in the limit $\left(N_{1}, N_{2}\right) \rightarrow \infty$ in such a way that $\lim _{N_{1} \rightarrow \infty} N_{2} / N_{1}=m$, for some $m>0$. The following theorem holds:

Theorem 3.1. Given $\mathbf{N}=\left(N_{1}, N_{2}\right)$, we can consider the $M C$ with transition probabilities (2.7), depending on the parameters $p_{k}=p_{k}\left(N_{k}\right), \Delta p_{k}=\Delta p_{k}\left(N_{k}\right), k=$ 1,2 and $\Delta p_{12}=\Delta p_{12}(\mathbf{N})$. Let us assume that $\lim _{N_{1} \rightarrow \infty} N_{2} / N_{1}=m$, for some $m>0$ and suppose that the following limits exist:

$$
\left\{\begin{array}{l}
\beta_{k}=\lim _{N_{k} \rightarrow \infty} N_{k}\left(p_{k}-\Delta p_{k}\right), \\
\alpha_{k}=\lim _{N_{k} \rightarrow \infty} \frac{1}{2} N_{k}\left(1-2 \Delta p_{k}\right), \quad k=1,2 \\
\gamma=\lim _{N_{2} \rightarrow \infty} 2 N_{2} \Delta p_{12} .
\end{array}\right.
$$

Then, the process $\chi^{\mathrm{N}}(t)$ with values in $K_{\mathbf{N}}$, defined by (3.5), converges as $N\left(=N_{1}\right)$ goes to infinity to the two-dimensional diffusion process $\chi(t)$ on $[0,1]^{2}$, whose associated backward differential equation has the form:

$$
\begin{aligned}
\frac{\partial q}{\partial t}\left(x_{1}, x_{2}, t\right) & =\sum_{i=1}^{2} b_{i}\left(x_{1}, x_{2}\right) \frac{\partial q}{\partial x_{i}}\left(x_{1}, x_{2}, t\right)+\frac{1}{2} \sum_{i, j}^{2} a_{i j}\left(x_{1}, x_{2}\right) \frac{\partial^{2} q}{\partial x_{i} \partial x_{j}}\left(x_{1}, x_{2}, t\right) \\
& =(L q)\left(x_{1}, x_{2}, t\right),\left(x_{1}, x_{2}, t\right) \in[0,1]^{2} \times R^{+}
\end{aligned}
$$

where

$$
\left\{\begin{array}{l}
b_{1}\left(x_{1}, x_{2}\right)=b_{1}\left(x_{1}\right)=\beta_{1}-2 \alpha_{1} x_{1} \\
b_{2}\left(x_{1}, x_{2}\right)=\frac{\beta_{2}}{m}+\frac{\gamma}{m} x_{1}-2 \frac{\alpha_{2}}{m} x_{2} \\
a_{i j}\left(x_{1}, x_{2}\right)=0, i \neq j \\
a_{11}\left(x_{1}, x_{2}\right)=x_{1}\left(1-x_{1}\right) \\
a_{22}\left(x_{1}, x_{2}\right)=\frac{1}{m} x_{2}\left(1-x_{2}\right)
\end{array}\right.
$$


with conditions

$$
\left\{\begin{array}{l}
0<\beta_{1}<2 \alpha_{1} \\
\beta_{2}+\gamma<2 \alpha_{2}, \gamma \geq 0 .
\end{array}\right.
$$

Roughly speaking, the above theorem says that, if we take:

$$
\left\{\begin{array}{l}
p_{k}=p_{k}\left(N_{k}\right)=\frac{1}{2}+\frac{\beta_{k}-\alpha_{k}}{N_{k}}+o\left(\frac{1}{N_{k}}\right) \\
\Delta p_{k}=\Delta p_{k}\left(N_{k}\right)=\frac{1}{2}-\frac{\alpha_{k}}{N_{k}}+o\left(\frac{1}{N_{k}}\right), \quad k=1,2 \\
\Delta p_{12}=\frac{\gamma}{2 N_{2}}+o\left(\frac{1}{N}\right)
\end{array}\right.
$$

for $N$ large, the rescaled MC approximates a continuous diffusion process on $[0,1]^{2}$.

Before giving the proof of Theorem 3.1, we need to introduce further notations and some lemmas whose proofs will be given later. Indeed, the proofs of Theorem 3.1 and lemmas are very similar to those given in Ref. 3 for the diffusion approximation of the MC relative to the model without hierarchy.

The rescaled two-dimensional MC $\chi^{\mathbf{N}}$ takes on values in $K_{\mathbf{N}}$ which we rewrite as $K_{\mathrm{N}}=\left\{\left(u_{1}, u_{2}\right)=\left(\left[x_{1} N_{1}\right] / N_{1},\left[x_{2} N_{2}\right] / N_{2}\right),\left(x_{1}, x_{2}\right) \in[0,1]^{2}\right\}([z]$ denotes the integer part of $z)$; its marginal distributions conditioned to the values at time $n$, that is to say, conditioned to the event

$$
\mathcal{G}_{n}=\left\{\left(1 / N_{1}\right) X^{N_{1}}(n)=u_{1},\left(1 / N_{2}\right) Y^{N_{2}}(n)=u_{2}\right\}
$$

are such that:

$$
\left\{\begin{array}{l}
\frac{1}{N_{1}} X^{N_{1}}(n+1) \mid \mathcal{G}_{n} \sim \frac{1}{N_{1}} Z_{N_{1}} \\
\frac{1}{N_{2}} Y^{N_{2}}(n+1) \mid \mathcal{G}_{n} \sim \frac{1}{N_{2}} Z_{N_{2}}
\end{array}\right.
$$

where $Z_{N_{1}} \sim B\left(N_{1}, p_{1}-\Delta p_{1}+2 \Delta p_{1} u_{1}\right)$ and $Z_{N_{2}} \sim B\left(N_{2}, p_{2}-\Delta p_{2}+2 \Delta p_{2} u_{2}+\right.$ $\left.2 \Delta p_{12} u_{1}\right), p .=p .(N),. \Delta p .=\Delta p .(N)$, where $B(n, \alpha)$ denotes the binomial distribution with parameters $n$ and $\alpha$.

Lemma 3.2. With the above notations, positive constants $c_{1}, c_{2}$ exist, such that:

$$
E_{x_{1}, x_{2}}\left(\left|Z_{N_{1}}-u_{1} N_{1}\right|^{4}\right) \sim c_{1} x_{1}^{2} N_{1}^{2}
$$

and

$$
E_{x_{1}, x_{2}}\left(\left|Z_{N_{2}}-u_{2} N_{2}\right|^{4}\right) \sim c_{2} x_{2}^{2} N_{2}^{2}
$$

where $E_{x_{1}, x_{2}}$ denotes the conditional expectation given $\chi^{\mathbf{N}}(n)=\left(x_{1}, x_{2}\right)$. By the relation $U(n) \sim r n^{2}$, we mean $\lim _{n \rightarrow \infty} U(n) / n^{2}=r$. 
Lemma 3.3. Denote $P_{\mathbf{N}}(\cdot, \cdot)$ the transition probability function of $\chi^{\mathbf{N}}$ and set:

$$
\begin{gathered}
b_{i, N}\left(x_{1}, x_{2}\right)=b_{i, N}\left(u_{1}, u_{2}\right)=N \int_{\|\mathbf{v}-\mathbf{u}\| \leq r}\left(v_{i}-u_{i}\right) P_{\mathbf{N}}(\mathbf{u}, \mathbf{d} \mathbf{v}), \\
a_{i j, N}\left(x_{1}, x_{2}\right)=a_{i j, N}\left(u_{1}, u_{2}\right)=N \int_{\|\mathbf{v}-\mathbf{u}\| \leq r}\left(v_{i}-u_{i}\right)\left(v_{j}-u_{j}\right) P_{\mathbf{N}}(\mathbf{u}, \mathbf{d} \mathbf{v})
\end{gathered}
$$

where $u_{1}=\left(1 / N_{1}\right)\left[x N_{1}\right], u_{2}=\left(1 / N_{2}\right)\left[y N_{2}\right], \mathbf{u}=\left(u_{1}, u_{2}\right), \mathbf{v}=\left(v_{1}, v_{2}\right) \in K_{\mathbf{N}}$.

Then $\forall \delta>0, \forall r>0$ :

$$
\begin{aligned}
& \text { (i) } \sup _{x_{1}^{2}+x_{2}^{2} \leq r^{2}} N P_{\mathbf{N}}(\mathbf{u},\{\mathbf{v}:\|\mathbf{v}-\mathbf{u}\|>\delta\}) \longrightarrow 0 \text {, as } N \rightarrow \infty, \\
& \text { (ii) } \sup _{x_{1}^{2}+x_{2}^{2} \leq r^{2}}\left|b_{i, N}\left(x_{1}, x_{2}\right)-b_{i}\left(x_{1}, x_{2}\right)\right| \longrightarrow 0 \text { as } N \rightarrow \infty, \\
& \text { (iii) } \sup _{x_{1}^{2}+x_{2}^{2} \leq r^{2}}\left|a_{i j, N}\left(x_{1}, x_{2}\right)-a_{i j}\left(x_{1}, x_{2}\right)\right| \longrightarrow 0 \text { as } N \rightarrow \infty \text {. }
\end{aligned}
$$

Proof of Theorem 3.1. The proof is quite analogous to that of Theorem 3.1 in Ref. 3. Indeed, as easily seen (cf. e.g. Ref. 9) (i), (ii), (iii) imply:

$$
\lim _{N \rightarrow \infty} \sup _{\mathbf{u} \in K_{\mathbf{N}}}\left|N\left(T^{\mathbf{N}}-I\right) f\left(u_{1}, u_{2}\right)-L f\left(u_{1}, u_{2}\right)\right|=0 \forall f \in C^{2}\left([0,1]^{2}\right),
$$

where $L$ is the differential operator defined in (3.7), and $T^{\mathbf{N}}$ is the associated transition semigroup, i.e. $T^{\mathbf{N}} f(\mathbf{u})=\int f(\mathbf{v}) P_{\mathbf{N}}(\mathbf{u}, \mathbf{d v})$. By using (3.18) and standard results, ${ }^{7}$ Theorem 3.1 follows.

Proof of Lemma 3.2. We omit the details of the proof, since it is exactly the same as that of Lemma 3.2 of Ref. 3 ; it suffices to replace $N$ and $E_{x}$ in Ref. 3 with $N_{1}$ and $E_{x_{1}, x_{2}}$, respectively.

\section{Proof of Lemma 3.3.}

(i) We have:

$$
\begin{aligned}
& P_{N}(u,\{v:\|v-u\|>\delta\}) \\
& \quad \leq P_{N_{1}}^{1}\left(u_{1},\left\{\left|v_{1}-u_{1}\right|>\delta / \sqrt{2}\right\}\right)+P_{N_{2}}^{2}\left(u_{2},\left\{\left|v_{2}-u_{2}\right|>\delta / \sqrt{2}\right\}\right),
\end{aligned}
$$

where $P_{N_{i}}^{i}(\cdot, \cdot)$ are the marginal transition probabilities. By the Chebyshev inequality:

$$
\begin{array}{r}
N P_{N_{i}}^{i}\left(u_{i},\left|v_{i}-u_{i}\right|>\delta / \sqrt{2}\right)=N P\left(\left|\frac{1}{N_{i}} Z_{N_{i}}-u_{i}\right|>\delta / \sqrt{2}\right) \\
\leq N \frac{E\left(\left|Z_{N_{i}} / N_{i}-u_{i}\right|^{4}\right)}{(\delta / \sqrt{2})^{4}}=4 N /\left(\delta^{4} N_{i}^{4}\right) E\left(\left|Z_{N_{i}}-N_{i} u_{i}\right|^{4}\right)
\end{array}
$$


By Lemma 3.2, the last quantity is of order $1 / N$, so by using (3.19), Lemma 3.3 follows.

(ii) The estimate of $b_{i, N}\left(x_{1}, x_{2}\right), i=1,2$ as $N \rightarrow \infty$, can be obtained exactly in the same way as was done in the proof of (ii) of Lemma 3.3 in Ref. 3, with the obvious modifications. Then, (3.16) follows easily.

(iii) Also the estimate of $a_{i j, N}\left(x_{1}, x_{2}\right), i, j=1,2$ as $N \rightarrow \infty$, can be obtained as in Lemma 3.3 of Ref. 3. The only thing to be remarked is that, for $i \neq j$, $a_{i, j, N}\left(x_{1}, x_{2}\right) \rightarrow 0$ as $N \rightarrow \infty$, i.e. the diffusion matrix is diagonal; this follows from the fact that the infinitesimal covariance is zero, thanks to the conditional independence of the processes $X_{1}(n)=X_{n}$ and $X_{2}(n)=Y_{n}$. Thus, (3.17) follows easily.

To the diffusion process, $\chi(t)$ is associated to the stochastic differential equation in $[0,1]^{2}$, having $b_{i}\left(x_{1}, x_{2}\right)$ as drift terms and $a_{i j}$ as diffusion matrix, that is $\chi(t)$ is the solution of

$$
\left\{\begin{array}{l}
d X_{1}=\left(\beta_{1}-2 \alpha_{1}\right) d t+\sqrt{X_{1}\left(1-X_{1}\right)} d W_{1}(t) \\
d X_{2}=\frac{1}{m}\left(\beta_{2}-2 \alpha_{2} X_{2}+\gamma X_{1}\right) d t+\sqrt{X_{2}\left(1-X_{2}\right)} d W_{2}(t) \\
X_{1}(0)=\bar{x}_{1}, X_{2}(0)=\bar{x}_{2}
\end{array}\right.
$$

where $W_{1}(t)$ and $W_{2}(t)$ are independent standard Brownian motions; the parameters are required to satisfy the following conditions:

$$
\beta_{i}>0, \quad i=1,2, \quad \gamma \geq 0, \quad 2 \alpha_{1}-\beta_{1}>0,2 \alpha_{2}-\beta_{2}-\gamma>0
$$

(3.21) are only technical conditions to assure that the process never exits from $[0,1]^{2}$. By Theorem 3.1, one can obtain information about the behavior of the MC; in particular, by means of the stationary measure of the continuous diffusion process, one can study the behavior of the MC, at infinite time.

When the cross-coupling capacity $\Delta p_{s w}$ is zero (this corresponding to $\gamma=0$, in the diffusion limit), the density of the stationary distribution for the continuous diffusion process can be explicitly found ${ }^{4}$ in the form of a product of two beta functions:

$u_{\alpha, \beta, 0}(x, y)=$ const $\cdot x^{2 \beta_{1}-1}(1-x)^{4 \alpha_{1}-2 \beta_{1}-1} y^{2 \beta_{2}-1}(1-y)^{4 \alpha_{2}-2 \beta_{2}-1}, \quad(x, y) \in[0,1]^{2}$.

For small, positive values of $\gamma$, the density $u_{\alpha, \beta, \gamma}(x, y)$ of the stationary measure is close to $u_{\alpha, \beta, 0}(x, y)$; by increasing $\gamma, u_{\alpha, \beta, \gamma}(x, y)$ gradually deviates from $u_{\alpha, \beta, 0}(x, y)$ (see Sec. 5 for more details).

From the diffusion approximation, the estimate for the stationary probabilities follows, for $N_{1}$ large:

$$
\Pi_{(i, j)} \approx \frac{1}{N_{1} N_{2}} u_{\alpha, \beta, \gamma}\left(\frac{i}{N_{1}}, \frac{j}{N_{2}}\right)
$$




\subsection{Attainability and unattainability of the boundary states}

Here, we discuss the possibility that the boundary states are assumed by the MC, in the dependence of the parameters. Indeed, the MC takes values $\left(n_{s}, n_{w}\right) \in$ $\left\{0,1, \ldots, N_{s}\right\} \times\left\{0,1, \ldots, N_{w}\right\}$, or, in other words, on the rectangle $\mathcal{R}=Z^{2} \cap$ $\left[0, N_{s}\right] \times\left[0, N_{w}\right]$ of the two-dimensional integer lattice. We are interested in stating conditions under which the boundary of $\mathcal{R}$ may (or may not) be reachable by the process.

Let $l_{0}$ be the portion of the boundary of $\mathcal{R}$ consisting of the states of type $\left(0, n_{w}\right)$ (situation of full uncoupling of strong bonds) and let $l_{1}$ be the portion of the boundary of $\mathcal{R}$ consisting of the states of type $\left(N_{s}, n_{w}\right)$ (situation of full coupling of strong bonds).

Moreover, let $\mathcal{L}_{0}$ and $\mathcal{L}_{1}$ be, respectively, the portion of boundary of $\mathcal{R}$ consisting of the states of type $\left(n_{s}, 0\right)$ (full uncoupling of weak bonds), and of type $\left(n_{s}, 1\right)$ (full coupling of weak bonds).

We recall that, for a process $\mathbf{X}$, a boundary point $\mathbf{x}$ is called attainable (or accessible) if $\mathbf{X}$ reaches $\mathbf{x}$ at a finite time, with positive probability, and unattainable (or inaccessible) otherwise. More generally, $\mathbf{x}$ is called attractive if $\mathbf{X}$ reaches $\mathbf{x}$ with positive probability, after a time which can be either finite or infinite; $\mathbf{x}$ is called repelling (or natural), otherwise.

Then, in the range of the parameters $p_{i}, \Delta p_{i}$ close to $1 / 2$ and $\Delta p_{s w}$ close to zero, by using the continuous diffusion approximation for $N_{1}=N_{s} \rightarrow \infty$, sufficient conditions can be found, so that each of the above portions of the boundary of $\mathcal{R}$ may (or may not) be reached, in the approximation $N_{s}$ large. Indeed, translating some results of Ref. 4 in terms of parameters $p_{i}=p_{i}\left(N_{i}\right), \Delta p_{i}=\Delta p_{i}\left(N_{i}\right), i=s, w$ and $\Delta p_{s w}=\Delta p_{s w}\left(N_{s}, N_{w}\right)$, in the approximation $N_{i} \rightarrow \infty$, we obtain the following properties.

Proposition 3.2. Let us assume that the parameters are taken in the range where the diffusion approximation holds; then for $N_{s}$ and $N_{w}$ large:

(i) $l_{0}$ is accessible and attractive if $2 N_{s}\left(p_{s}-\Delta p_{s}\right)<1$, inaccessible and repelling otherwise;

(ii) $l_{1}$ is accessible and attractive if $2 N_{s}\left(1-p_{s}-\Delta p_{s}\right)<1$, inaccessible and repelling otherwise;

(iii) $\mathcal{L}_{0}$ is accessible and attractive if $2 N_{w}\left(p_{w}-\Delta p_{w}+2 \Delta p_{s w}\right)<1$, and it is inaccessible and repelling if $2 N_{w}\left(p_{w}-\Delta p_{w}\right) \geq 1$;

(iv) $\mathcal{L}_{1}$ is accessible and attractive if $2 N_{w}\left(1-p_{w}-\Delta p_{w}\right)<1$, and it is inaccessible and repelling if $2 N_{w}\left(1-p_{w}-\Delta p_{w}-2 \Delta p_{s w}\right) \geq 1$.

These results satisfactorily agree with the numerical simulations (see Sec. 5).

\subsection{The Poisson approximation (for $p_{k}, \Delta p_{k}$ not necessarily close to} 1/2)

The diffusion limit obtained in Sec. 3.4 concerns the approximation of the MC with transition probabilities $(2.7)$ to a continuous diffusion process in $[0,1]^{2}$, when 
$N_{k} \rightarrow \infty$, in the case when $p_{k}, \Delta p_{k}$ are close to $1 / 2$ and $\Delta p_{s w}$ is close to zero. Now, we look for an asymptotic expression of the transition probabilities for $N_{w} / N_{s} \sim m$ and $N_{s}$ large, in the case when all the parameters can assume any value far from $1 / 2$. In this situation, the MC is approximated by a Poisson process as $N_{s} \rightarrow \infty$.

This approximation is suitable for low cooperativity proteins, where $\Delta p_{k}$ are close to zero and therefore it is complementary to the diffusion approximation. In this situation, the $\mathrm{MC}$ is approximated, as $N_{s} \rightarrow \infty$, by a Poisson process.

Let us consider a sequence of MCs, where $p_{k}=p_{k}(N), \Delta p_{k}=\Delta p_{k}(N), \Delta p_{s w}=$ $\Delta p_{s w}(N), k=s, w$, and let us suppose that there exist constants $\lambda_{k}, \mu_{k}$ and $\rho$ such that, as $N_{s} \rightarrow \infty$,

$$
\left\{\begin{array}{l}
N_{k}\left(p_{k}-\Delta p_{k}\right) \rightarrow \mu_{k}, \\
2 \Delta p_{k} \rightarrow \lambda_{k} \\
2 m \Delta p_{s w} \rightarrow \rho .
\end{array}\right.
$$

Then, let us consider the first three factors in (2.7) (for simplicity we write $p, \Delta p$, $i, j, N$ in place of $p_{s}, \Delta p_{s}, i_{s}, j_{s}, N_{s}$, respectively):

$$
\begin{aligned}
& \left(\begin{array}{c}
N \\
j
\end{array}\right)\left(p-\Delta p+\frac{2 \Delta p}{N} i\right)^{j}\left[1-\left(p-\Delta p+\frac{2 \Delta p}{N} i\right)\right]^{N-j} \\
= & \frac{N(N-1) \cdots(N-j+1)}{j !}\left(p-\Delta p+\frac{2 \Delta p}{N} i\right)^{j}\left[1-\frac{N(p-\Delta p)+2 \Delta p i}{N}\right]^{N-j} .
\end{aligned}
$$

Now, as $N \rightarrow \infty$

$$
\begin{gathered}
\frac{(2 \Delta p i+N(p-\Delta p))^{j}}{\left(1-\frac{N(p-\Delta p)+2 \Delta p i}{N}\right)^{j}} \rightarrow\left(2 \Delta p i+\mu_{s}\right)^{j} \\
{\left[1-\frac{N(p-\Delta p)+2 \Delta p i}{N}\right]^{N} \rightarrow e^{-\left(\mu_{s}+2 \Delta p i\right)}}
\end{gathered}
$$

Therefore

$$
\begin{aligned}
I_{i, j} & :=\left(\begin{array}{c}
N \\
j
\end{array}\right)\left((p-\Delta p)+\frac{2 \Delta p}{N} i\right)^{j}\left[1-\left((p-\Delta p)+\frac{2 \Delta p}{N} i\right)\right]^{N-j} \\
& \rightarrow \frac{1}{j !}\left(2 \Delta p i+\mu_{s}\right)^{j} e^{-\left(2 \Delta p i+\mu_{\theta}\right)} .
\end{aligned}
$$

Since as $N_{s} \rightarrow \infty, 2 \Delta p \longrightarrow \lambda_{s}$, then

$$
I_{i, j} \longrightarrow \frac{1}{j !}\left(\lambda_{s} i+\mu_{s}\right)^{j} e^{-\left(\lambda_{s} i+\mu_{s}\right)} .
$$


Thus, for the first component of the process, we have:

$$
\begin{aligned}
& \mathbf{P}\left(S_{[N t]}\left(\xi^{k+1}\right)=j \mid S_{[N t]}\left(\xi^{k}\right)=i\right) \\
& =\left(\begin{array}{c}
{[N t]} \\
j
\end{array}\right)\left((p-\Delta p)+\frac{2 \Delta p}{[N t]} i\right)^{j}\left[1-\left((p-\Delta p)+\frac{2 \Delta p}{[N t]} i\right)\right]^{[N t]-j} \\
& \quad \rightarrow \frac{1}{j !}\left(\lambda_{s} i+\mu_{s} t\right)^{j} e^{-\left(\lambda_{s} i+\mu_{s} t\right)} .
\end{aligned}
$$

Let us consider a Poisson process $\left(X_{t}\right)_{t \geq 0}$ with density $\lambda_{s} i+\mu_{s} t$, i.e.

$$
\begin{aligned}
& \mathbf{P}\left(X_{t}=j \mid X_{0}=i\right)=\frac{1}{j !}\left(\lambda_{s} i+\mu_{s} t\right)^{j} e^{-\left(\lambda_{s} i+\mu_{s} t\right)}, \\
& \mathbf{P}_{t, N, i}(A):=\mathbf{P}\left(A \mid S_{[N, t]}\left(\xi^{k}\right)=i\right), \\
& \tilde{\mathbf{P}}_{t, N, i}:=\mathbf{P}_{t, N, i} \circ\left(S_{[N t]}\left(\xi^{k+1}\right)\right) .
\end{aligned}
$$

Then, as $N=N_{s} \rightarrow \infty$

$$
\tilde{\mathbf{P}}_{t, N, i} \rightarrow \mathbf{P} \circ X_{t}^{-1}
$$

or equivalently $\forall r \in \mathbf{N}$

$$
\tilde{\mathbf{E}}_{t, N, i}\left[\left(S_{[N t]}\left(\xi^{k+1}\right)\right)^{r}\right] \rightarrow \mathbf{E}\left(X_{t}^{r}\right) .
$$

Turning to the asympotic expression for the transition probabilities, in an analogous way, we can estimate the other factors in (2.7). Thus, finally we obtain, as $N_{s} \rightarrow \infty$

$$
\begin{aligned}
P_{\left(i_{s}, i_{w}\right)\left(j_{s}, j_{w}\right)} \sim & \frac{1}{j_{s} !}\left(\lambda_{s} i_{s}+\mu_{s}\right)^{j_{s}} e^{-\left(\lambda_{s} i_{s}+\mu_{s}\right)} \\
& \times \frac{1}{j_{w} !}\left(\lambda_{w} i_{w}+\mu_{w}+\rho i_{s}\right)^{j_{w}} e^{-\left(\lambda_{w} i_{w}+\mu_{w}+\rho i_{s}\right)} .
\end{aligned}
$$

We remark that (3.24) holds in the approximation

$$
\left\{\begin{array}{l}
\Delta p_{k} \sim \frac{\lambda_{k}}{2} \\
p_{k} \sim \frac{\lambda_{k}}{2}+\frac{\mu_{k}}{N_{k}} \\
\Delta p_{s w} \sim \frac{\rho}{2 m}
\end{array}\right.
$$

\section{Parameters Estimation}

In the range of parameters where the diffusion approximation of the process $\left(X_{n}, Y_{n}\right)$ holds (see Sec. 3.3), $p_{i}, \Delta p_{i}, i=s, w, \Delta p_{s w}$ can be estimated by using the maximum likelihood method. Indeed, the maximum likelihood method can be directly 
applied to the MC to estimate parameters, but the point at which the likelihood function, obtained by the transition probabilities (2.7), takes its maximum cannot be analytically found. For the same reason, the Poisson approximation is not convenient to estimate parameters either. Instead, the likelihood function obtained by using the diffusion approximation is analytically tractable, thanks to its exponential form.

We recall from Sec. 3.3 that the evolution of the diffusion process is described by the following stochastic differential equation:

$$
\left\{\begin{array}{l}
d X=\left(\beta_{1}-2 \alpha_{1}\right) d t+\sqrt{X(1-X)} d W_{1}(t) \\
d Y=\frac{1}{m}\left(\beta_{2}-2 \alpha_{2} Y+\gamma X\right) d t+\sqrt{Y(1-Y)} d W_{2}(t) \\
X(0)=\bar{X}, Y(0)=\bar{Y}
\end{array}\right.
$$

where $W_{1}(t)$ and $W_{2}(t)$ are independent standard Brownian motions.

By discretization of Eq. (4.1), one obtains:

$$
\left\{\begin{array}{l}
x_{n+1}=x_{n}+\left(\beta_{1}-2 \alpha_{1} x_{n}\right) h+\sqrt{x_{n}\left(1-x_{n}\right)} \Delta W_{1}, \\
y_{n+1}=y_{n}+\frac{1}{m}\left(\beta_{2}-2 \alpha_{2} y_{n}+\gamma x_{n}\right) h+\sqrt{y_{n}\left(1-y_{n}\right)} \Delta W_{2}, \\
x_{0}=\bar{x}, \quad y_{0}=\bar{y},
\end{array}\right.
$$

where $x_{n}$ and $y_{n}, n=0,1, \ldots, M$ denote, respectively, the two processes evaluated at the time $t_{n}=n h$, and $\Delta W_{i}=W_{i}\left(t_{n+1}\right)-W_{i}\left(t_{n}\right), i=1,2$.

Equation (4.2) means that the two-dimensional random variable $\left(\begin{array}{c}X_{n+1} \\ Y_{n+1}\end{array}\right)$, conditionally to $\left(X_{n}=x_{n}, Y_{n}=y_{n}\right)$ is distributed according to a bivariate Gaussian with expectation vector

$$
\left(\begin{array}{c}
x_{n}+\left(\beta_{1}-2 \alpha_{1} x_{n}\right) h \\
y_{n}+\frac{1}{m}\left(\beta_{2}-2 \alpha_{2} y_{n}+\gamma x_{n}\right) h
\end{array}\right)
$$

and covariance matrix

$$
\left(\begin{array}{cc}
x_{n}\left(1-x_{n}\right) h & 0 \\
0 & y_{n}\left(1-y_{n}\right) h
\end{array}\right)
$$

From (4.4), given the finite sequence $\left(x_{n}, y_{n}\right)_{n=0,1, \ldots, M}$, we obtain the likelihood function:

$$
\begin{aligned}
L\left(\alpha_{i}, \beta_{i}, \gamma\right) & \\
= & \prod_{n=1}^{M} \frac{1}{2 \pi h \sqrt{x_{n} y_{n}\left(1-x_{n}\right)\left(1-y_{n}\right)}} \exp -\frac{\left[x_{n+1}-x_{n}-\left(\beta_{1}-2 \alpha_{1} x_{n}\right) h\right]^{2}}{2 h x_{n}\left(1-x_{n}\right)} \\
& \times \exp -\frac{\left[y_{n+1}-y_{n}-\frac{1}{m}\left(\beta_{2}-2 \alpha_{2} y_{n}+\gamma x_{n}\right) h\right]^{2}}{2 h y_{n}\left(1-y_{n}\right)}
\end{aligned}
$$


as well as the logarithm of the likelihood function:

$$
\begin{aligned}
V\left(\alpha_{i}, \beta_{i}, \gamma\right)= & -M \log (2 \pi h)-\frac{1}{2} \sum_{n=1}^{M}\left\{\log \left(x_{n}\left(1-x_{n}\right)\right)+\log \left(y_{n}\left(1-y_{n}\right)\right)\right\} \\
& -\frac{1}{2} \sum_{n}\left\{\frac{\left(x_{n+1}-x_{n}-\beta_{1} h+2 \alpha_{1} x_{n} h^{2}\right.}{h x_{n}\left(1-x_{n}\right)}\right. \\
& \left.+\frac{\left(y_{n+1}-y_{n}-\beta_{2} h+2 \alpha_{2 y_{n}} h-\gamma x_{n} h\right)^{2}}{h y_{n}\left(1-y_{n}\right)}\right\}
\end{aligned}
$$

The maximum likelihood estimates $\hat{\alpha}_{i}, \hat{\beta}_{i}, \hat{\gamma}$ of the parameters $\alpha_{i}, \beta_{i}, i=1,2$ and $\gamma$ are obtained by setting to zero the partial derivatives of the function $V(\cdot)$ with respect to its arguments. In this way, we obtain the system:

$$
\left\{\begin{array}{l}
\frac{\partial V}{\partial \alpha_{1}}=-2 \sum_{n=1}^{M} \frac{x_{n+1}-x_{n}-\beta_{1} h+2 \alpha_{1} x_{n} h}{1-x_{n}}=0 \\
\frac{\partial V}{\partial \beta_{1}}=\sum_{n=1}^{M} \frac{x_{n+1}-x_{n}-\beta_{1} h+2 \alpha_{1} x_{n} h}{x_{n}\left(1-x_{n}\right)}=0 \\
\frac{\partial V}{\partial \alpha_{2}}=-2 \sum_{n=1}^{M} \frac{y_{n+1}-y_{n}-\beta_{2} h+2 \alpha_{2} y_{n} h-\gamma x_{n} h}{1-y_{n}}=0 \\
\frac{\partial V}{\partial \beta_{2}}=\sum_{n=1}^{M} \frac{y_{n+1}-y_{n}-\beta_{2} h+2 \alpha_{2} y_{n} h-\gamma x_{n} h}{y_{n}\left(1-y_{n}\right)}=0 \\
\frac{\partial V}{\partial \gamma}=\sum_{n=1}^{M} \frac{\left(y_{n+1}-y_{n}-\beta_{2} h+2 \alpha_{2} y_{n} h-\gamma x_{n} h\right) x_{n}}{y_{n}\left(1-y_{n}\right)}=0 .
\end{array}\right.
$$

A very long, but straightforward calculation shows that the solution of the above system is given by:

$$
\left\{\begin{array}{l}
\hat{\alpha}_{1}=\frac{1}{2 h} \frac{C D-E A}{A^{2}-C B} \\
\hat{\beta}_{1}=\frac{1}{h A}\left(D+\frac{B(C D-E A)}{A^{2}-C B}\right), \\
\hat{\alpha}_{2}=\frac{1}{2 h \Delta}\left(-E^{\prime} A^{\prime} \Delta_{3}-D^{\prime} \Delta_{2}^{2}-E^{\prime \prime} C^{\prime} \Delta_{1}+E^{\prime} \Delta_{1} \Delta_{2}+E^{\prime \prime} \Delta_{2} A^{\prime}+\Delta_{3} C^{\prime} D^{\prime}\right) \\
\hat{\beta}_{2}=-\frac{1}{h \Delta}\left(-D^{\prime} A^{\prime} \Delta_{3}-E^{\prime \prime} B^{\prime} \Delta_{2}-E^{\prime} \Delta_{1}^{2}+E^{\prime \prime} A^{\prime} \Delta_{1}+D^{\prime} \Delta_{1} \Delta_{2}+E^{\prime} B^{\prime} \Delta_{3}\right) \\
\hat{\gamma}=-\frac{1}{h \Delta}\left(-A^{\prime 2} E^{\prime \prime}-E^{\prime} B^{\prime} \Delta_{2}-D^{\prime} C^{\prime} \Delta_{1}+D^{\prime} A^{\prime} \Delta_{2}+E^{\prime} \Delta_{1} A^{\prime}+E^{\prime \prime} B^{\prime} C^{\prime}\right),
\end{array}\right.
$$


where

$$
\left\{\begin{array}{l}
A=\sum_{n} \frac{1}{1-x_{n}} \\
B=\sum_{n} \frac{x_{n}}{1-x_{n}} \\
C=\sum_{n} \frac{1}{x_{n}\left(1-x_{n}\right)} \\
D=\sum_{n} \frac{x_{n+1}-x_{n}}{1-x_{n}} \\
E=\sum_{n} \frac{x_{n+1}-x_{n}}{x_{n}\left(1-x_{n}\right)} \\
A^{\prime}=\sum_{n} \frac{1}{1-y_{n}} \\
B^{\prime}=\sum_{n} \frac{y_{n}}{1-y_{n}}, \\
C^{\prime}=\sum_{n} \frac{1}{y_{n}\left(1-y_{n}\right)} \\
D_{1}^{\prime}=\sum_{n} \frac{y_{n+1}-y_{n}}{1-y_{n}} \\
\Delta_{2}=\sum_{n} \frac{x_{n}}{1-y_{n}}, \\
E^{\prime}=\sum_{n} \frac{y_{n+1}-y_{n}}{y_{n}\left(1-y_{n}\right)} \\
E_{n}=\sum_{n} \frac{x_{n}\left(y_{n+1}-y_{n}\right)}{y_{n}\left(1-y_{n}\right)} \\
y_{n}\left(1-y_{n}\right) \\
\Delta_{2} \Delta_{2}^{2}+C^{\prime} \Delta_{1}^{2}-2 A^{\prime} \Delta_{1} \Delta_{2}-C^{\prime} B^{\prime} \Delta_{3}
\end{array}\right.
$$

Finally, from $\hat{\alpha}_{i}, \hat{\beta}_{i}, \hat{\gamma}$, the estimates $\hat{p}_{i}, \hat{\Delta} p_{i}$ and $\hat{\Delta} p_{12}$ of the parameters $p_{i}, \Delta p_{i}$, $i=1,2$ and $\Delta p_{12}$ are easily obtained by using (3.9), in the approximation $N_{1}=$ $N_{s}, N_{2}=N_{w}$ large. 


\section{Numerical Results and Computer Simulations}

In this section, we deal with numerical results obtained via computer simulations. A Monte-Carlo method was used to simulate the evolution of the system. The joint stationary probabilities were found numerically, by computing the frequencies of visit to each state $(i, j) \in\left\{0,1, \ldots, N_{s}\right\} \times\left\{0,1, \ldots, N_{w}\right\}$ in a very long simulation run (from $10^{5}$ to $10^{6}$ time iteration steps in some cases), for several values of the

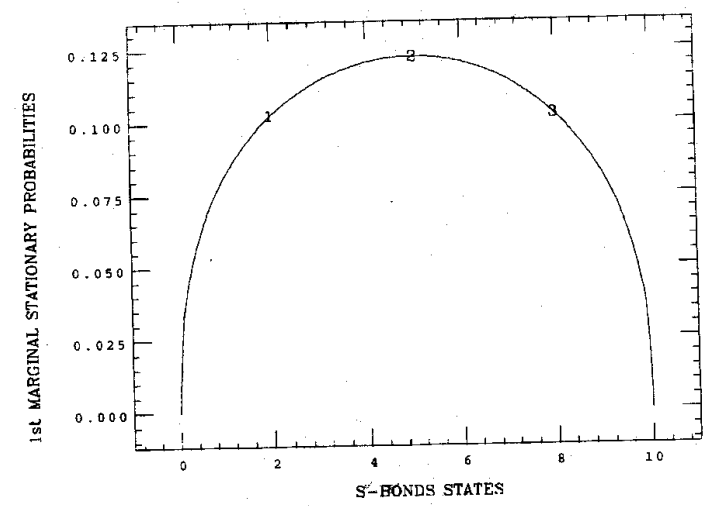

(a)

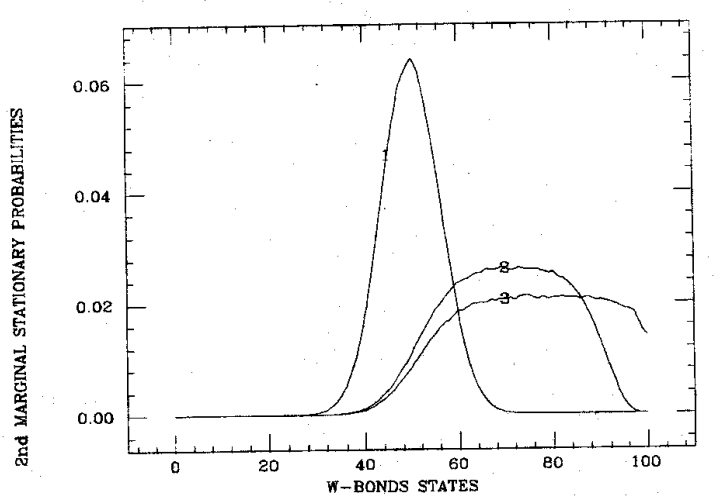

(b)

Fig. 1. (a) Plots of first marginal stationary distributions as a function of the $s$-bonds state (i.e. the number of strong bonds which are formed at infinite time) relative to the following three cases: $p_{s}=p_{w}=0.5, \Delta p_{s}=0.45, \Delta p_{w}=0.3$, and $\Delta p_{s w}=0,0.08$ and 0.1 for (1), (2) and (3) respectively. Notice that the curves overlap, since the change in the cross-coupling capacity does not affect the bonds of strong type. The computation has required a simulation run of one million iteration steps. (b) Comparison of plots of second marginal stationary distributions versus the $w$-bonds state (i.e. the number of weak bonds which are formed at infinite time) in the three cases relative to (a). 


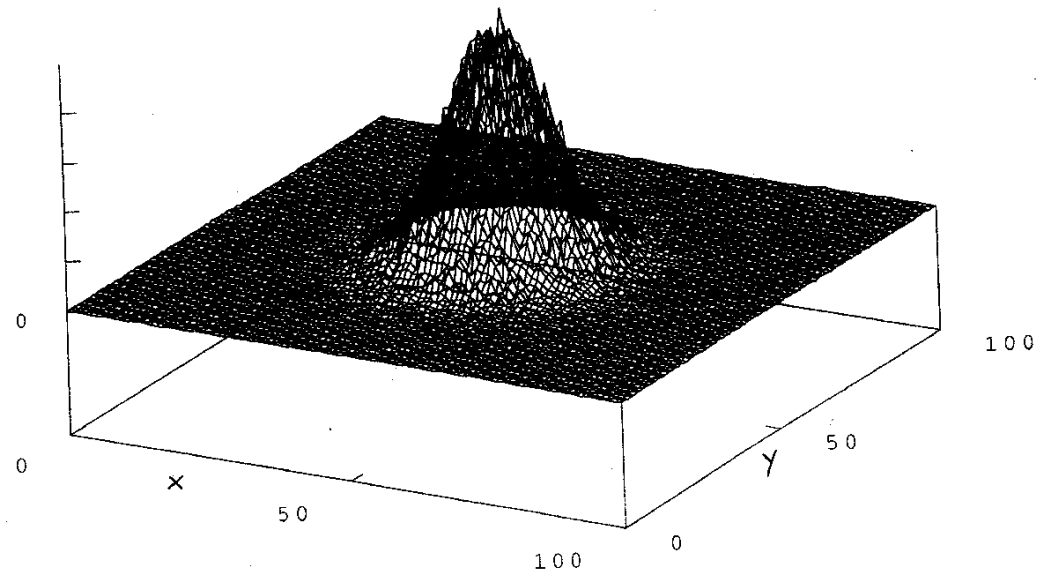

(a)

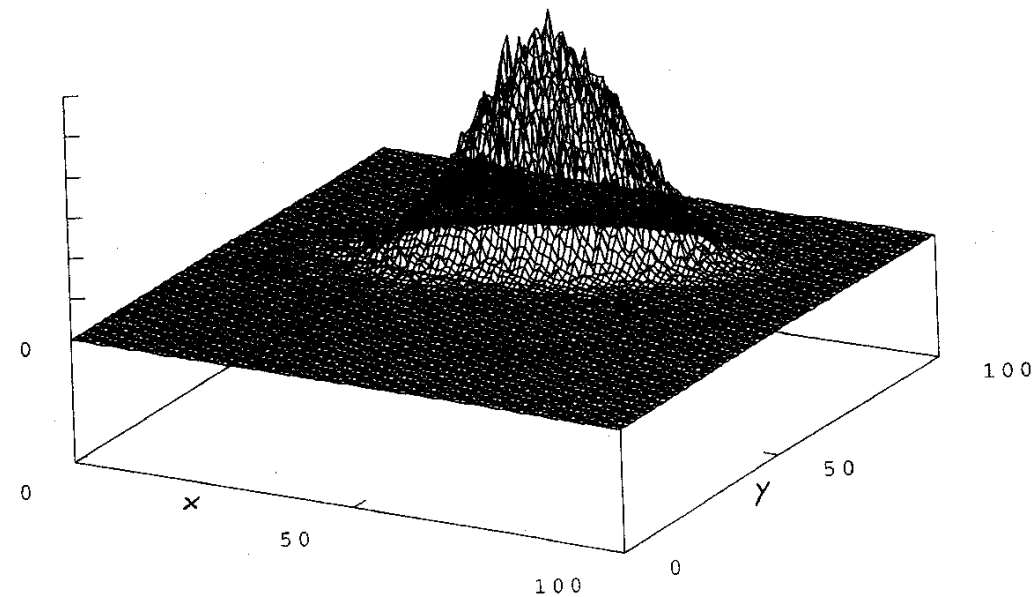

(b)

Fig. 2. (a) Three-dimensional plot of the joint stationary distribution for $N_{s}=N_{w}=100$, $p_{s}=p_{w}=0.5, \Delta p_{s}=\Delta p_{w}=0.4, \Delta p_{s w}=0$. The peak value is obtained exactly at the middle, $\left(x_{M}, y_{M}\right)=(50,50)$. For these values of the parameters, with probability one, no portion of the boundary of $\mathcal{R}$ is accessible. The computation required a simulation run of 100,000 iteration (b) Thrimensional plot of the joint stationary distribution for $p_{s}=p_{w}=0.5, \Delta p_{s}=$ $0.45, \Delta p_{w}=0.2, \Delta p_{s w}=0.1$. On the $x$-axes the $s$-bonds state is reported, from 0 to $N_{s}$; on the , $y$-axes the $w$-bonds state system stays at the state $(x, y)$ at equilibrium, i.e. $z=\Pi_{(x, y)}$. Here, for the probability that the system stays at the state (x,y) at easily seen, the frequency of visit to a state sake of diagram simplicity, we took $N_{s}=N_{w}=100$ (as easily seen, the freque value at $\left(x_{M}, y_{M}\right)$, does not depend on the maximum number of the allowe is shifted in the direction of the $y$-axes, i.e. the most probable value, is not the middle state, but it station run of 100,000 iteration steps. with $\left(x_{M}, y_{M}\right) \approx(50,70)$. The computation required a sima (c) As in (b) for $N_{s}=N_{w}=100, p_{s}=p_{w}=0.5, \Delta p_{s}=0.1, \Delta p_{w}$, but the peak is more narrow than point is obtained approximately at the same values of $x, y$ of $(b)$, but the $\left(x_{M}, y_{M}\right)$. The computation in (b); the distribution is more concentrated around
required a simulation run of 100,000 iteration steps. 


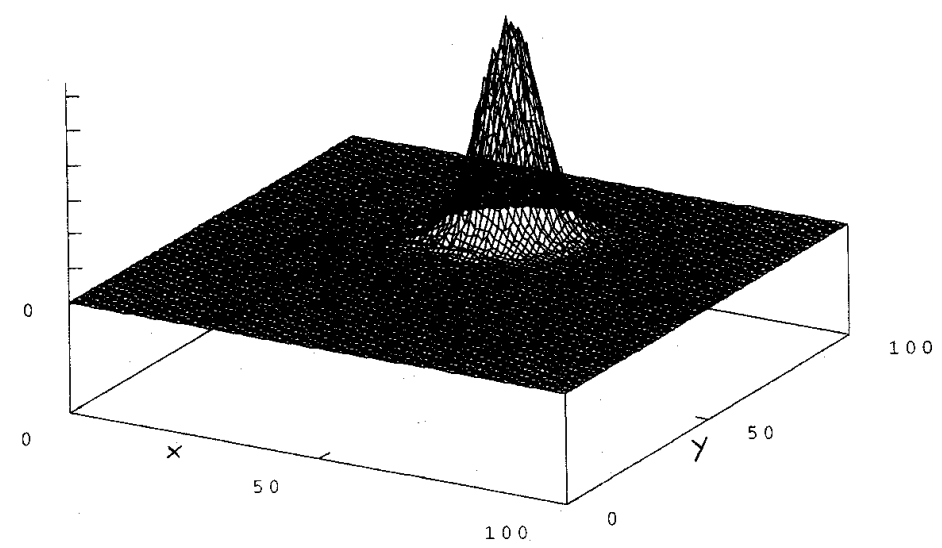

(c)

Fig. 2. (Continued)

parameters. Also the marginal stationary probabilities were computed, for both. processes $X_{n}$ and $Y_{n}$.

In Figs. 1a-b the plots of the marginal stationary probabilities are reported as a function of the state $n_{s} \in\left\{0,1, \ldots, N_{s}\right\}$ and $n_{w} \in\left\{0,1, \ldots, N_{w}\right\}$, respectively, for a set of values of the parameters (see the figures' caption).

From these graphs it follows that, for zero values of the cross-coupling capacity $\Delta p_{s w}$, the curves relative to the marginal stationary probabilities behave like beta functions centered at the middle state. When $\Delta p_{s w}$ increases from 0 to positive values, the curve relative to the first marginal stationary probabilities does not change, while the abscissa of the maximum of the curve relative to weak bonds (second marginal stationary distribution) shifts to the right. This means, as expected, that, for $\Delta p_{s w}>0$, the influence of strong bonds on the formation of additional weak ones becomes non-negligible.

In fact, for $\Delta p_{s w}>0$, not only the mean of the second marginal distribution is shifted to the right, but also there are very few values concentrated around the mean, implying that, with a large probability, a number of $w$-type bonds, belonging to an interval of length $30 \% N_{w}$ around the mean, can be formed at equilibrium (see Fig. 1b). All that is detectable also by examination of three-dimensional plots of the joint stationary distribution of the process $\left(X_{n}, Y_{n}\right)$ (see Figs. 2a-c). Moreover, the simulations show that, at parity of $p_{s}, p_{w}$ and $\Delta p_{s w}$ the two parameters $\Delta p_{s}$ and $\Delta p_{w}$ can be interchanged, in some sense, if one wants to obtain the same behavior in the formation of weak bonds. This means that the mean value of $w$-type bonds at equilibrium remains unchanged under the condition that a decrease of $\Delta p_{w}$ is accompanied by an increase of $\Delta p_{s}$ (see Figs. 3a-b).

Thus, we observe an effective picture of a cooperative phenomenon, with a hierarchic structure, where for $\Delta p_{s w} \neq 0$, the evolution of the system is 


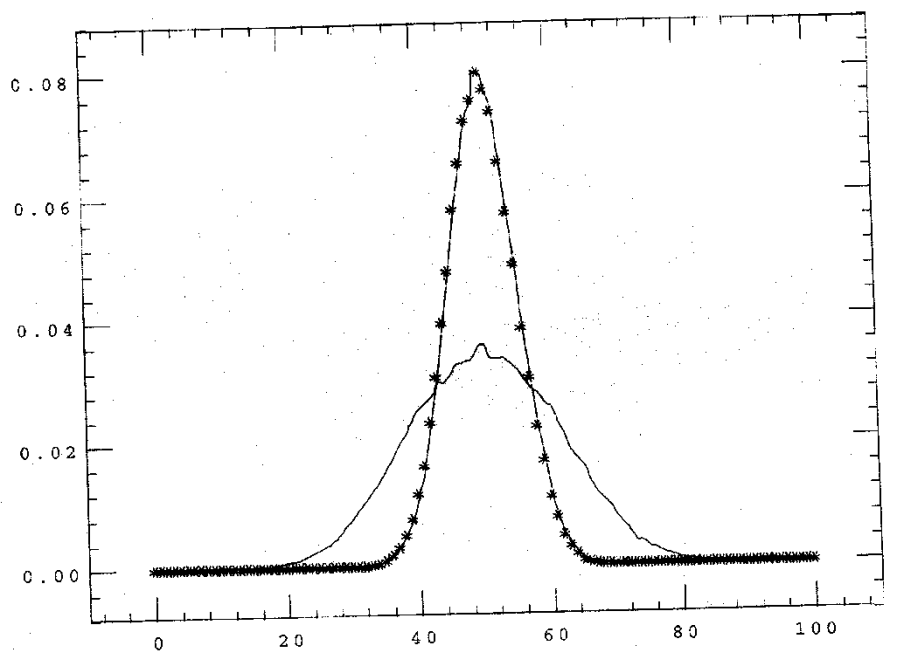

(a)

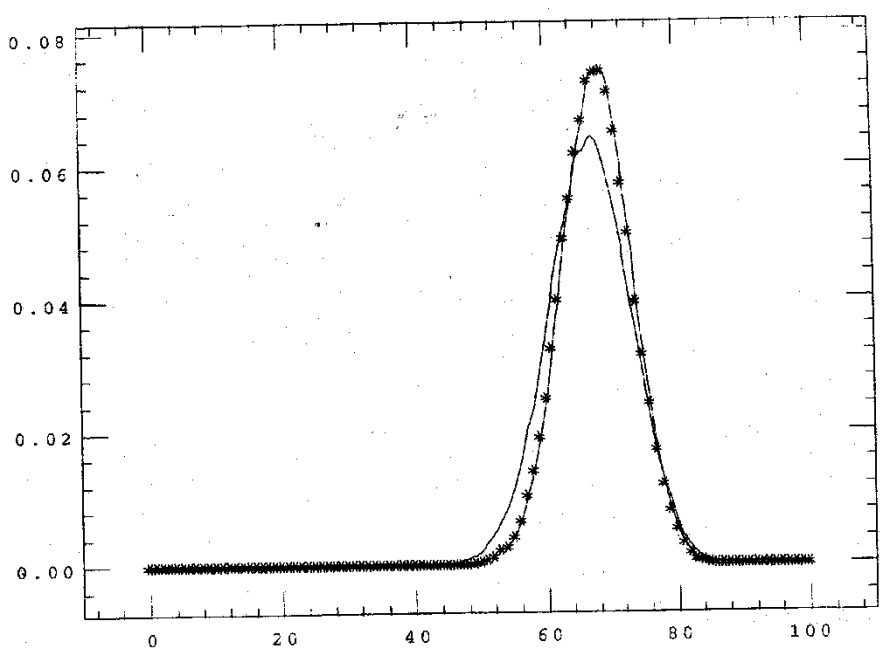

(b)

Fig. 3. (a) Comparison of plots of first marginal stationary distributions in the two cases relative to Figs. $2 \mathrm{~b}(---)$ and $2 \mathrm{c}(* * *)$. (b) Comparison of plots of second marginal stationary distribution in the two cases relative to Figs. $2 \mathrm{~b}(---)$ and $2 \mathrm{c}(* * *)$.

essentially driven by the capability to form strong bonds, since they also influence the formation of weak ones.

For what concerns attainability or unattainability of the boundary states (full coupling or uncoupling for one or both types of bonds), the results of Proposition 3.2 are amply confirmed by numerical computation.

The possibility or impossibility to reach the boundaries of $\mathcal{R}$ with a positive probability is shown by three-dimensional plots of the joint stationary distribution 


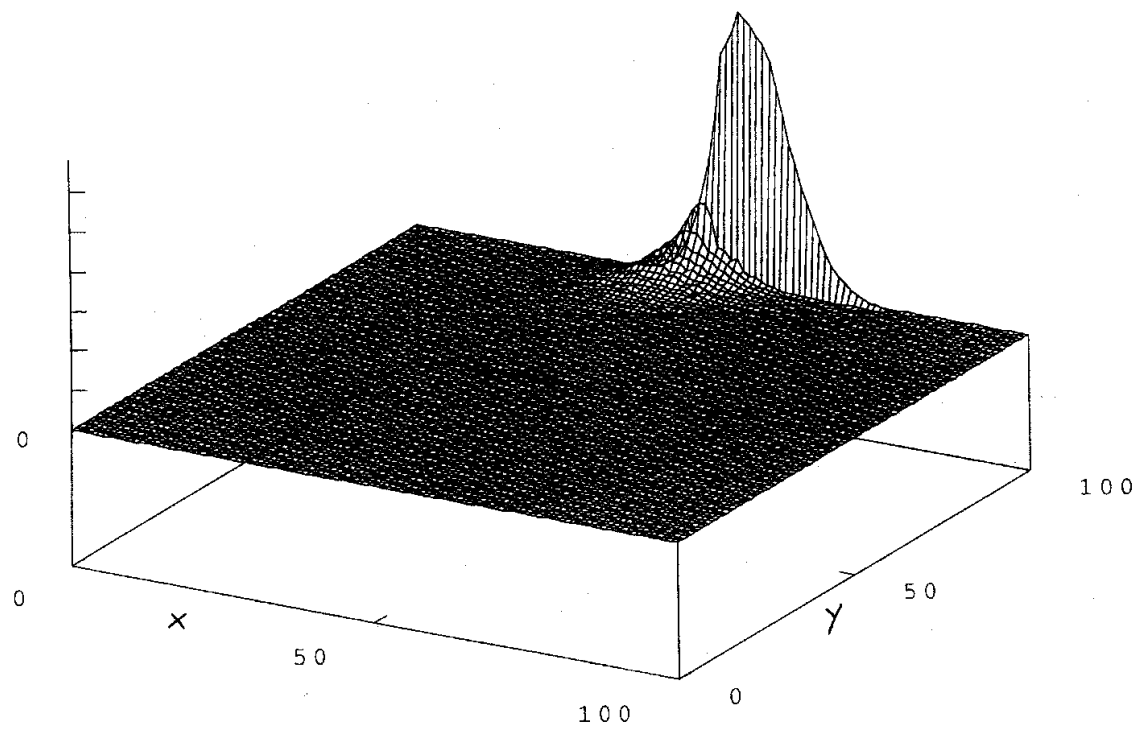

(a)

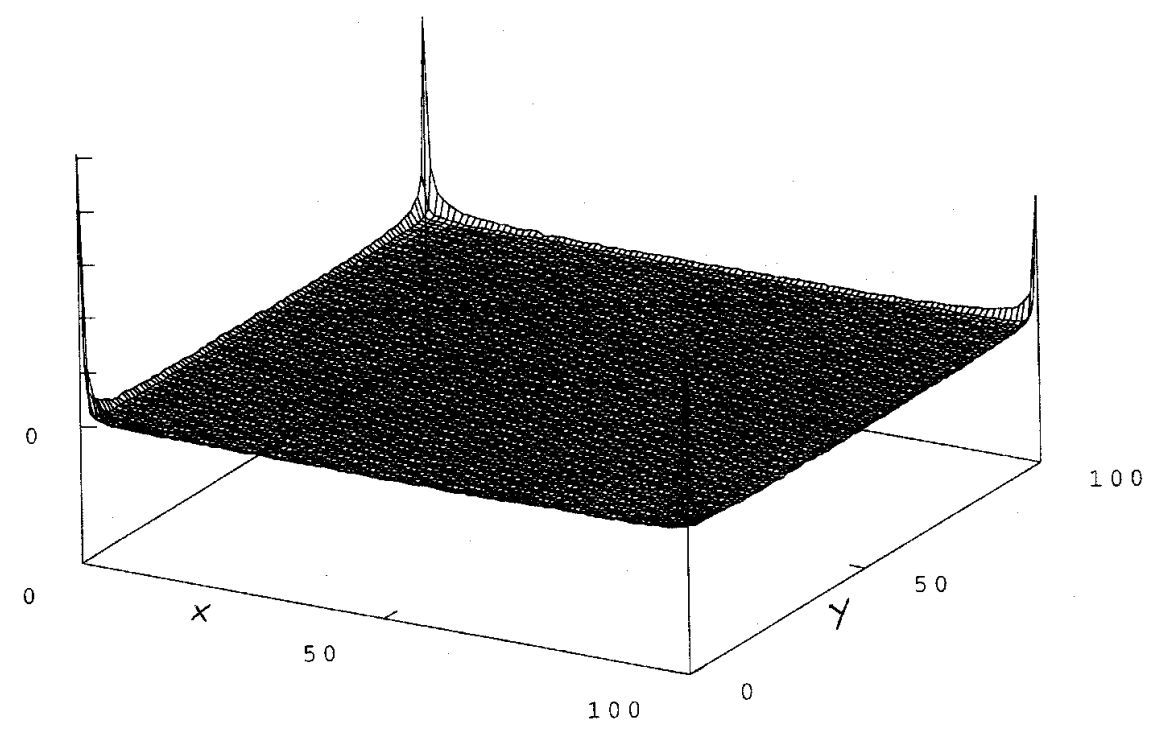

(b)

Fig. 4. (a) As in Fig. 2, for $p_{s}=p_{w}=0.5, \Delta p_{s}=\Delta p_{w}=0.3, \Delta p_{s w}=0.1$. The $y$-coordinate of the peak value is largely shifted to the right. (b) As in Fig. 2, for $p_{s}=p_{w}=0.5, \Delta p_{s}=$ $\Delta p_{w}=0.499, \Delta p_{s w}=0$; the graph is a concave surface. For these values of the parameters, all the portions of the boundary of $\mathcal{R}$ are attainable. 


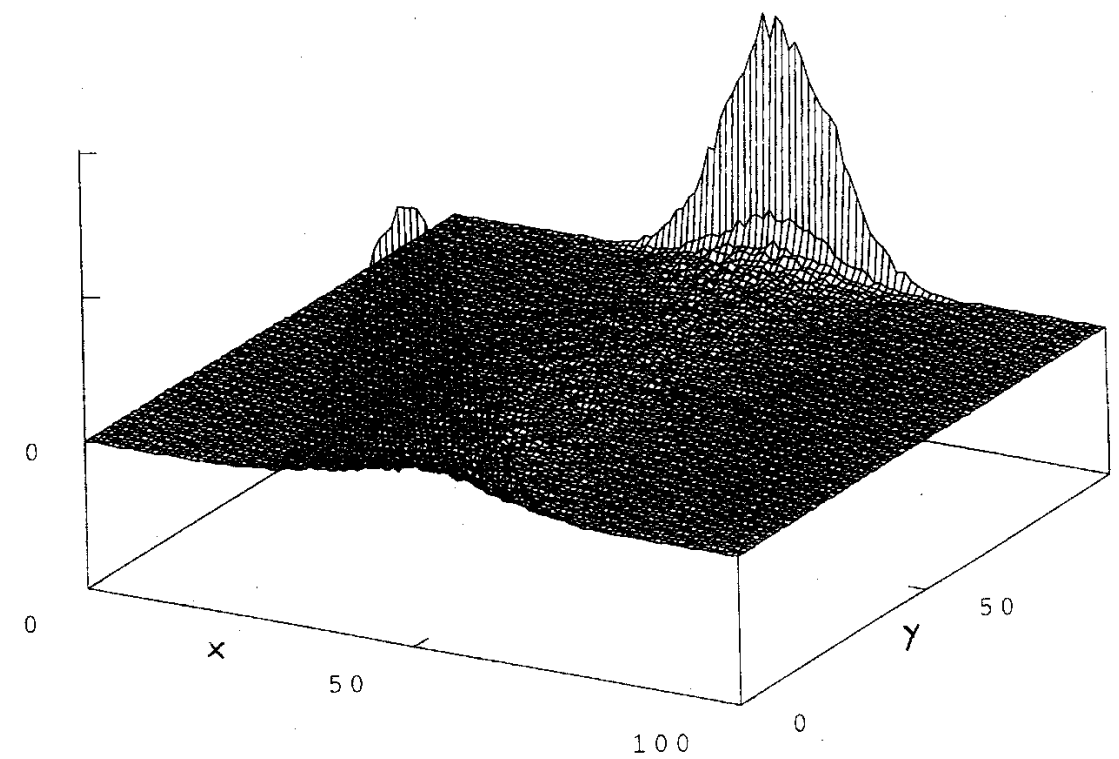

(a)

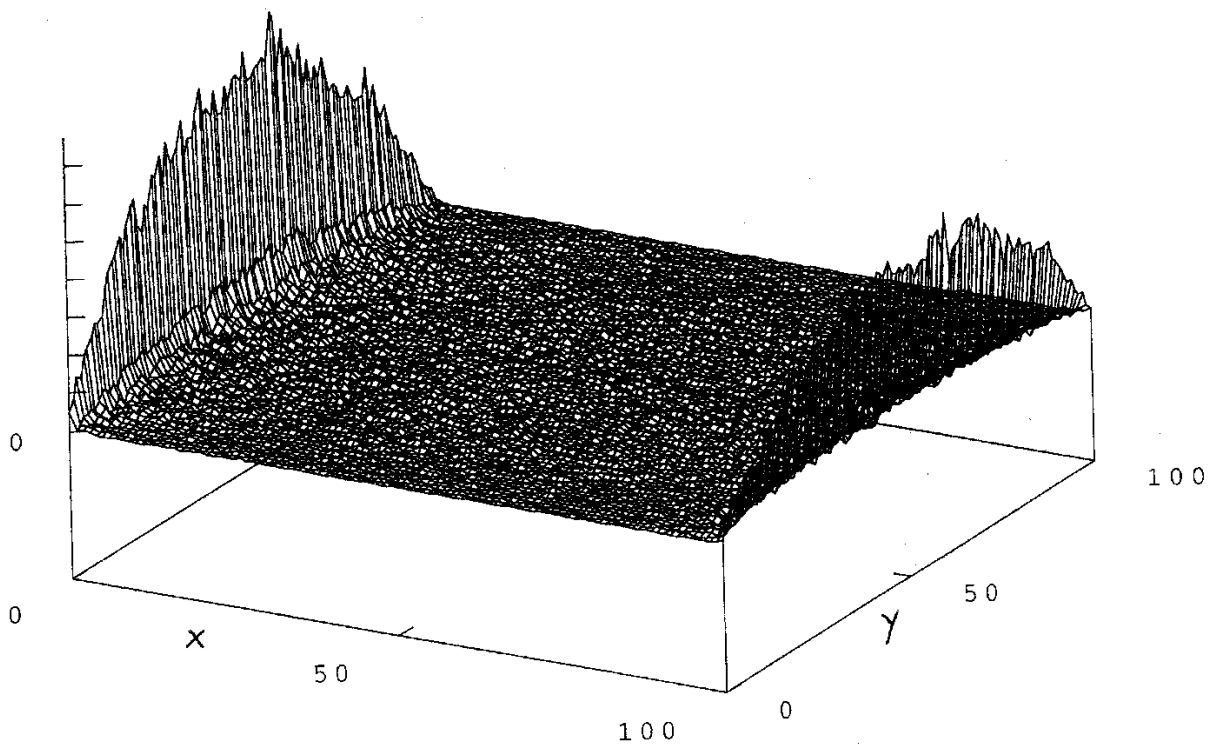

(b)

Fig. 5. (a) As in Fig. 2, for $p_{s}=p_{w}=0.5, \Delta p_{s}=0.4, \Delta p_{w}=0.499, \Delta p_{s w}=0$. For these values of the parameters, the portions of boundary $l_{0}$ and $l_{1}$ are inaccessible, while $\mathcal{L}_{0}$ and $\mathcal{L}_{1}$ are accessible. (b) As in Fig. 2, for $p_{s}=p_{w}=0.5, \Delta p_{s}=0.499, \Delta p_{w}=0.49, \Delta p_{s w}=0$. For these values of the parameters, the portions of boundary $l_{0}$ and $l_{1}$ are accessible, while $\mathcal{L}_{0}$ and $\mathcal{L}_{1}$ are inaccessible. 
of the process $\left(X_{n}, Y_{n}\right)$. In the case of Figs. 2a-c and $4 \mathrm{a}$, for instance, no portion of the boundary of $\mathcal{R}$ can be reached (roughly speaking, the probability density calculated at the boundary is zero).

Figure 4b illustrates the case in which each portion of the boundary of $\mathcal{R}$ can be reached (roughly speaking, the probability density is infinite at the boundary).

Figure 5a illustrates the case when the portions of the boundary $l_{0}$ and $l_{1}$ are inaccessible and $\mathcal{L}_{0}$ and $\mathcal{L}_{1}$ are accessible. Figure $5 \mathrm{~b}$ refers to the converse case in which $l_{0}$ and $l_{1}$ are accessible and $\mathcal{L}_{0}$ and $\mathcal{L}_{1}$ are inaccessible (see the figures caption).

Now, we are concerned with numerical results about parameters estimation. The parameters of the model were estimated by means of the maximum likelihood method.

\subsection{Parameters estimation by using simulated data}

By using sequences of simulated data, obtained via a Monte-Carlo method by running a FORTRAN computer program with given input values of the parameters, we recovered the estimates of these parameters, as if they were unknown. We used sequences consisting of 2,000 two-dimensional data, but equally good estimates can be obtained by using shorter sequences. The obtained estimates are very satisfactory, in a lot of cases. In fact, we obtained a high accuracy in the estimates of all the parameters (the error was less than $0.1 \%$ ) when the true (input) value of $\Delta p_{s w}$ is small enough $\left(<5 \times 10^{-2}\right)$ (see Table 1). When the input value of $\Delta p_{s w}$ is greater than $5 \times 10^{-2}$, the estimates of $p_{s}$ and $\Delta p_{s}, \Delta p_{w}$ are equally precise,

Table 1. Parameters estimation recovered by the maximum likelihood method, using several simulated data obtained from given input parameters. We used sequences consisting of 2,000 two-dimensional data $\left(x_{n}, y_{n}\right)_{n=1, \ldots, 2000}$.

Input Values

\begin{tabular}{|c|l|l|l|l|c|}
\hline$p_{s}$ & $\Delta_{p_{s}}$ & $p_{w}$ & $\Delta_{p_{w}}$ & $\Delta_{p_{s w}}$ & $p_{w}+\Delta_{p_{s w}}$ \\
\hline 0.5 & 0. & 0.5 & 0. & 0. & 0.5 \\
0.5 & 0.1 & 0.5 & 0.1 & 0.01 & 0.51 \\
0.5 & 0.1 & 0.5 & 0.1 & 0.05 & 0.55 \\
0.5 & 0.1 & 0.5 & 0.1 & 0.06 & 0.56 \\
0.5 & 0.1 & 0.5 & 0.1 & 0.1 & 0.6 \\
0.5 & 0.3 & 0.5 & 0.2 & 0.02 & 0.52 \\
0.5 & 0.3 & 0.5 & 0.3 & 0.03 & 0.53 \\
0.5 & 0.3 & 0.5 & 0.3 & 0.06 & 0.56 \\
0.5 & 0.3 & 0.5 & 0.3 & 0.08 & 0.58 \\
0.5 & 0.2 & 0.4 & 0.1 & 0.02 & 0.42 \\
0.5 & 0.4 & 0.5 & 0.4 & 0.05 & 0.55 \\
\hline
\end{tabular}


Table 1 (Continued)

Estimates

\begin{tabular}{|l|l|l|l|l|c|}
\hline$\hat{p}_{s}$ & $\hat{\Delta}_{p_{s}}$ & $\hat{p}_{w}$ & $\hat{\Delta}_{p_{w}}$ & $\hat{\Delta}_{P_{s w}}$ & $\hat{p}_{w}+\hat{\Delta}_{p_{s w}}$ \\
\hline 0.500 & 0.00 & 0.494 & 0.00 & 0.00 & 0.5 \\
0.500 & 0.10 & 0.495 & 0.099 & 0.015 & 0.51 \\
0.500 & 0.10 & 0.52 & 0.10 & 0.024 & 0.549 \\
0.500 & 0.10 & 0.528 & 0.102 & 0.0303 & 0.559 \\
0.500 & 0.10 & 0.56 & 0.100 & 0.037 & 0.599 \\
0.500 & 0.31 & 0.499 & 0.20 & 0.021 & 0.52 \\
0.500 & 0.31 & 0.498 & 0.30 & 0.030 & 0.53 \\
0.500 & 0.31 & 0.51 & 0.30 & 0.0412 & 0.557 \\
0.500 & 0.31 & 0.52 & 0.30 & 0.0543 & 0.575 \\
0.500 & 0.20 & 0.399 & 0.0889 & 0.0189 & 0.418 \\
0.500 & 0.40 & 0.508 & 0.40 & 0.0413 & 0.55 \\
\hline
\end{tabular}

while the error in the estimates of $p_{w}$ and $\Delta p_{s w}$ is bigger; however, the estimate of the sum $p_{w}+\Delta p_{s w}$, is more precise (the error is less than $0.01 \%$ ) (see Table 1). This is satisfactory enough, since, on average, one can consider that the ground probability to form a weak bond is given by the sum of two contributions: the one coming from the weak bonds themselves (i.e. $p_{w}$ ), and that due to the strong bonds (i.e. $\Delta p_{s w}$ ). Moreover, some inaccuracy has to be ascribed to the fact that the method for estimating parameters makes use of the diffusion approximation in the limit $N_{s}, N_{w} \rightarrow \infty$, while $N_{s}$ and $N_{w}$ are taken finite in the simulation.

\subsection{Parameters estimation for a real protein}

To test our model on a real system, we analyzed the data relative to a molecular dynamics simulation of the thermal denaturation of the protein Barnase, appeared in Ref. 5. In that paper the authors simulated the unfolding of the protein following a temperature jump from $300 \mathrm{~K}$ to $600 \mathrm{~K}$, at neutral pH. During the relaxation, the molecule rapidly expands and the total potential energy increases due to a progressive rupture of intermolecular interactions.

As a measure of the number of the strong and weak bonds of our model, we used the potential energy for the Coulomb (electrostatic) and van der Waals forces, respectively, considering intermolecular and protein-water interactions. To obtain an estimate of the number of bonds, an average energy associated to every bond was needed. We chose the values

$$
\begin{gathered}
\Delta E_{e s}=-1 \mathrm{kcal} / \mathrm{mole} \\
\Delta E_{v d w}=-0.1 \mathrm{kcal} / \mathrm{mole} .
\end{gathered}
$$


These values are somewhat arbitrary, but, reasonably, they do not vary more than a factor of 10; thus, the actual number of bonds should not be critical as long as it is large enough. Using the above values, we obtained the number of weak and strong bonds as a function of time (with a time-step of 1 ps), as shown in Figs 6a-b. We find $N_{s} \approx 7730, N_{w} \approx 10,350, N_{\text {tot }} \approx 18,080$.

The data corresponding to the total number of bonds (see Fig. 6c) were analyzed by the model for cooperative interactions without hierarchic structure. ${ }^{1} \mathrm{By}$ means of the one-dimensional diffusion approximation and the maximum likelihood method, we obtained the estimates:

$$
\bar{p}_{\text {tot }}=0.60, \bar{\Delta} p_{\text {tot }}=0.37 \text {. }
$$

In this case $\bar{\Delta} p_{\text {tot }}$ represents the global cooperativity of the protein.

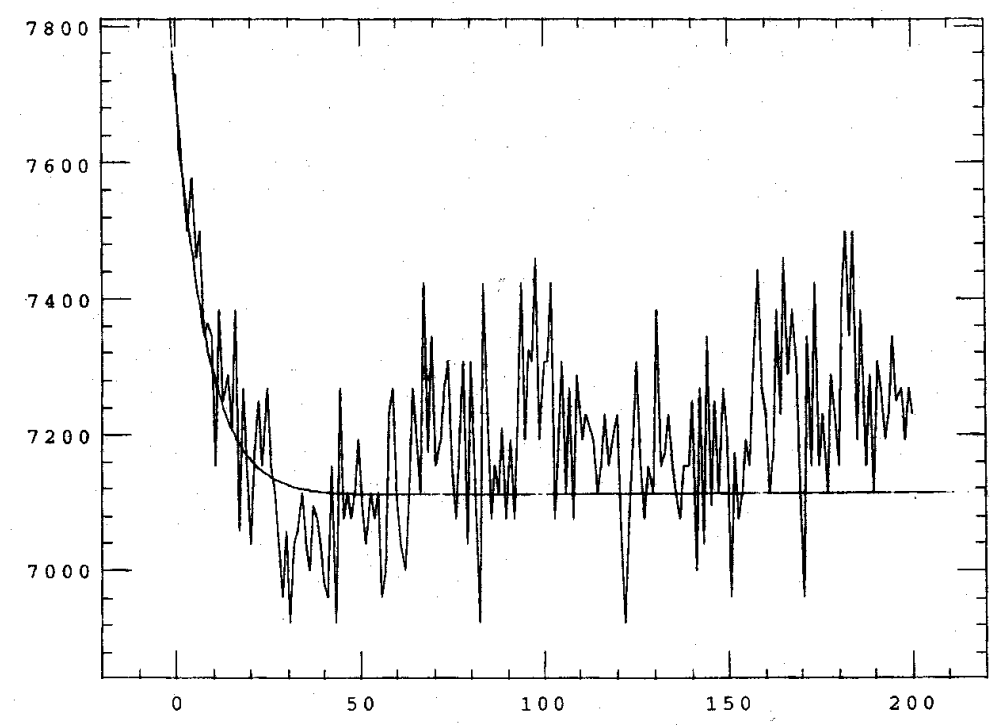

(a)

Fig. 6. (a) Plot of the time evolution (in ps) of the number of strong bonds obtained from the electrostatic energy for the thermal denaturation of the protein Barnase (data reported from Fig. $5 \mathrm{~g}$ of Ref. 5). From these data the parameters regarding the strong bonds of the model have been estimated (see text for the numerical values). The superimposed curve represents the time evolution of the average process obtained by different simulation runs with input parameters equal to these estimates. (b) Plot of the time evolution (in ps) of the number of weak bonds obtained from the van der Waals energy for the thermal denaturation of the protein Barnase (data reported from Fig. $5 f$ of Ref. 5). From these data and those of Fig. 6a, the parameters of the model regarding the weak bonds were estimated (see text for the numerical values). The superimposed curve represents the time evolution of the average process obtained by different simulation runs with input parameters equal to these estimates. (c) Plot of the time evolution (in ps) of the total number of bonds (strong + weak) for the protein Barnase. From these data, the parameters for the total number of bonds (strong + weak) were estimated (see text for the numerical values). The superimposed curve represents the time evolution of the average process. 


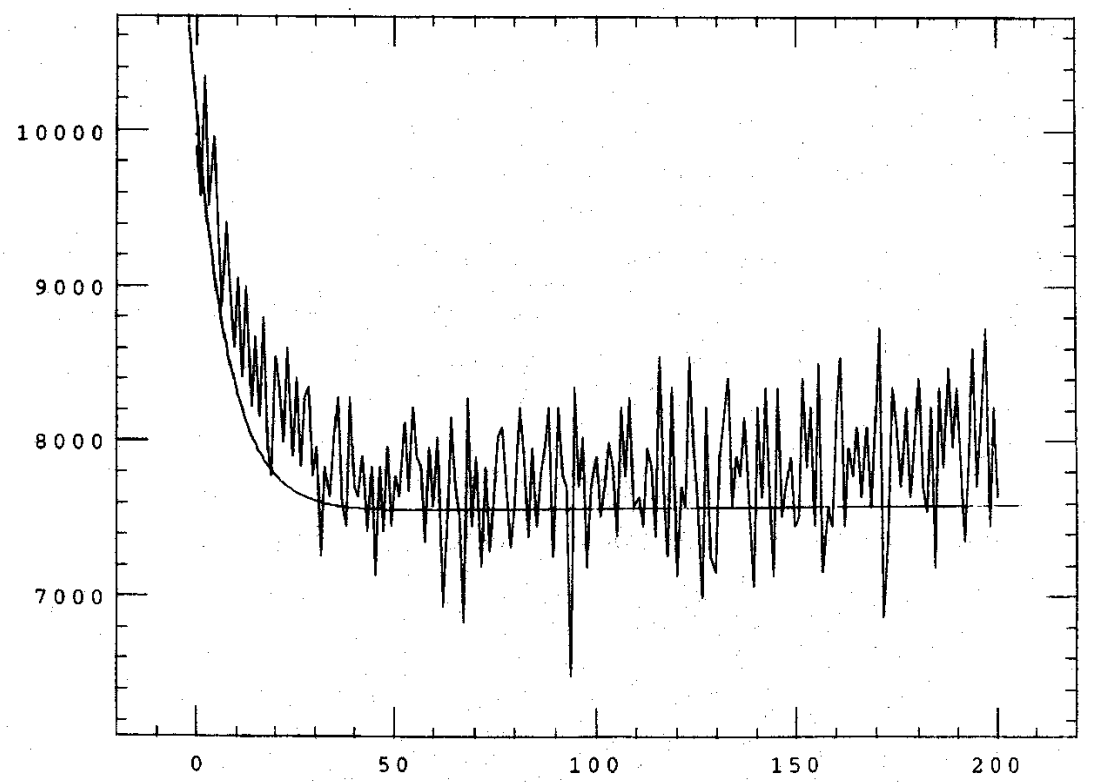

(b)

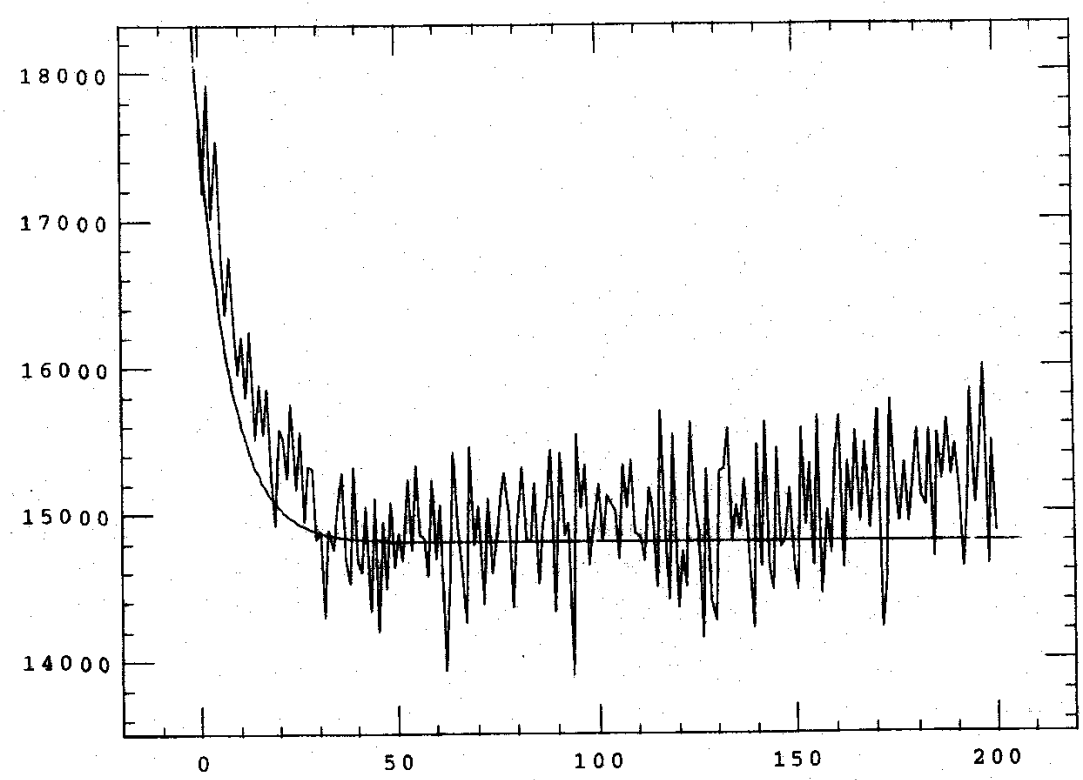

(c)

Fig. 6. (Continued) 
The two series of data corresponding to strong and weak bonds were analyzed by means of the hierarchic model; by using the two-dimensional diffusion approximation and the maximum likelihood method (see (4.7)), we obtained estimates for $\alpha_{i}$, $\beta_{i}$ and $\gamma$. From these, we recovered the estimates for $p_{i}, \Delta p_{i}$ and $\Delta p_{s w}$ :

$$
\left\{\begin{array}{l}
\bar{p}_{s}=0.67 \\
\bar{p}_{w}=0.33 \\
\bar{\Delta} p_{s}=0.25 \\
\bar{\Delta} p_{w}=0.16 \\
\bar{\Delta} p_{s w}=0.18
\end{array}\right.
$$

These values show a certain degree of cooperativity in both the strong and weak bonds and a dependence of the weak bonds on the strong ones.

As a further test of our model, we exchanged the roles of the van der Waals and electrostatic interactions, using the first ones as the strong bonds and the second ones as the weak ones. The fit obtained in this way is worse and $\Delta p_{s w}$ is smaller (of order $10^{-3}$ ) and even negative. This supports our choice of the electrostatic interactions as the strong bonds, and the significance of the value found for $\Delta p_{s w}$ in that case.

We also calculated the likelihood function directly from the transition probabilities of the MC (2.7); then, we searched for its maximum, by using a routine for global optimization with constraints. Putting $\Delta p_{s w}=\Delta p_{s}$ (this hypothesis is justified by the fact that the strong bonds influence the weak ones in the same extent at least as they influence themselves), we obtained the following estimates of the parameters:

$$
\left\{\begin{array}{l}
\tilde{p}_{s}=0.70 \\
\tilde{p}_{w}=0.25 \\
\tilde{\Delta} p_{s}=0.22 \\
\tilde{\Delta} p_{w}=0.18 \\
\tilde{\Delta} p_{s w}=0.22
\end{array}\right.
$$

In this way, we removed the restriction on the magnitude of the parameter $\Delta p_{s w}$, since the diffusion approximation is adapted in principle to describe only a situation in which $\Delta p_{s w}$ is rather small. However, we must observe that, also by using the diffusion approximation, the estimate of $\Delta p_{s w}$ is not much smaller than $\Delta p_{s}$. Moreover, to find the maximum of the likelihood function without using the diffusion approximation, we spent a much longer computer time than in the case of the diffusion approximation (in that case, the point at which the likelihood function takes the maximum can be analytically found).

Although there are some differences between numerical values of the estimates obtained with the two methods, the shapes of the average processes (obtained by 
different simulation runs with input parameters equal to the estimates respectively found) appear to be approximately the same. (See Fig. 6, where the data from Ref. 5 regarding the time-evolution of the number of bonds (strong, weak, and strong + weak) are reported together with the graphs of the three average processes, obtained by estimating the parameters indifferently with one of two methods.)

We remark that the estimates obtained by using the diffusion approximation in the case of protein interactions without hierarchic structure are still more satisfactory. This is because when the term $\Delta p_{s w}$ disappears, the problem about the range of the cross-coupling parameter also disappears.

In fact, in the case of data regarding the unfolding of the bovine pancreatic trypsin inhibitor (BPTI), obtained by Daggett and Levitt through molecular dynamic simulations, ${ }^{1,6}$ the estimates obtained by using the diffusion approximation are very close to those obtained numerically by us in our previous work, ${ }^{1}$ where these data were analyzed by means of the model for cooperative interactions in proteins without hierarchic structure. Precisely, up to an error of order $10^{-5}$, we recovered (by using the one-dimensional diffusion limit) the same values for $\bar{p}$ and $\bar{\Delta} p(\bar{p}=0.4956, \Delta p=0.4575$; cf. Fig. 13 in Ref. 1 and the relative discussion).

\section{Concluding Remarks}

In this paper, we described a Markovian model for the cooperative interactions in proteins, based on a hierarchic structure of the interactions. It relies on the arbitrary splitting of interactions into two classes, strong and weak, and tests the preponderance of one class on the other (a greater number of different types of classes could result only in a heavy notation).

The model generalizes the one valid for homogeneous cooperative interactions in protein molecules previously studied by the authors. ${ }^{1}$

The time evolution of the system has been studied as a function of five parameters, three of which are related to the cooperativity. The main assumption is that, when the polypeptidic chain is relaxing toward the native protein conformation, the amino acidic residues can be bounded by strong and weak interactions, and the strong interactions can influence the weak ones, but not vice versa; thus, the presence of a strong bond between amino acidic residues could facilitate the local formation of weak ones.

The model depends on parameters $p_{s}$ and $p_{w}$ (which represent the mean probability to form strong and weak bonds, respectively), on parameters $\Delta p_{s}$ and $\Delta p_{w}$ (which represent the cooperativity capacities for strong and weak bonds, respectively), and finally on the parameter $\Delta p_{s w}$ (which represents the cross coupling capacity). As in the case of interactions without structure, ${ }^{1}$ the model presents a surprising wealth of qualitative behaviors, when the five parameters are varied. Moreover, this stochastic model, which is alternative to deterministic ones, presents some advantages: (i) its simplicity, (ii) the fact that it depends only on five parameters, (iii) the relatively short time required for numerical simulations. 
The evolution of the system was described by a two-dimensional Markov chain with state space $\left\{(i, j): i \in\left\{0,1, \ldots, N_{s}\right\}, j \in\left\{0,1, \ldots, N_{w}\right\}\right\}$, where $N_{s}$ and $N_{w}$ are, respectively, the total numbers of permitted couplings among residues of strong and weak type. Then, absorption problems were studied as a function of the parameters. When $p_{i}>\Delta p_{i}>0, p_{i}+\Delta p_{i}<1,(i=s, w)$ and $p_{w}+\Delta p_{w}+$ $2 \Delta p_{s w}<1$, the chain is irreducible and the stationary probabilities exist (they are the probabilities that the system stays in given states, at equilibrium). By sinulating the evolution of the system with a Monte-Carlo method, and computing the frequency of visit to each state $(i, j) \in\left\{0,1, \ldots, N_{s}\right\} \times\left\{0,1, \ldots, N_{w}\right\}$ in a very long simulation run (about $10^{6}$ time iteration steps), we numerically found the stationary probabilities, for several values of the parameters. Also the marginal stationary probabilities were computed for each component of the two-dimensional process.

The numerical results show the particular relevance of the cross-coupling capacity parameter $\Delta p_{s w}$. When it is set to zero, the two-dimensional process has independent components, each of them behaves as in the case of protein interactions without hierarchic structure. When $\Delta p_{s w}$ increases from zero to a positive value, the first marginal stationary probabilities do not change, while the peak point of the joint stationary distribution is shifted to the right in the direction of the $y$-axes (number of weak bonds). This agrees with the fact that, as expected, for $\Delta p_{s w}>0$, the influence of strong bonds on the formation of additional weak ones becomes non-negligible. Moreover, the simulations show that, for fixed $p_{s}, p_{w}$ and $\Delta p_{s w}$ the two parameters $\Delta p_{s}$ and $\Delta p_{w}$ can be interchanged, in some sense, if one wants to obtain the same behavior in the formation of weak bonds. This means that the mean value of $w$-type bonds at equilibrium remains unchanged under the condition that a decrease of $\Delta p_{w}$ is accompanied by an increase of $\Delta p_{s}$.

For what concerns attainability or unattainability of the boundary states (full coupling or uncoupling for one or both types of bonds), the possibility or impossibility to reach the boundary states can be detected by the three-dimensional plot of the stationary probabilities: roughly speaking, a portion of the boundary is accessible or inaccessible, whether or not the stationary distribution is infinite, or zero at that portion.

Indeed, the qualitative results described above can be obtained by studying the diffusion approximation of the system. In fact, for $p_{i}$ and $\Delta p_{i}(i=s, w)$ close to $1 / 2$ and $\Delta p_{s w}$ close to zero, the discrete process can be approximated, for large $N_{s}$ and $N_{w}$, by a continuous diffusion process. Once such a description of the system is available, its evolution could be more easily studied by considering the associated stochastic differential equation for the continuous process. Actually, it can be shown that the stationary distribution has a density and its shape is that of a perturbed product of two beta functions (the larger $\Delta p_{s w}$, the more it deviates from the product of beta functions), and this agrees with numerical results. The stationary probabilities can be approximately obtained by (3.11). We estimated the parameters of the model by using the maximum likelihood method 
and the diffusion approximation. The estimates re-obtained from simulated data are rather precise, and excellent in a lot of cases. For the present model, the diffusion approximation represents (to our knowledge) the only analytical tool to obtain parameters estimation.

To test our model on a real system, we analyzed the data relative to a molecular dynamics simulation of the thermal denaturation of the protein Barnase, appeared in Ref. 5. In that paper, during the relaxation following a temperature jump from $300 \mathrm{~K}$ to $600 \mathrm{~K}$, the total potential energy increases due to a progressive rupture of intermolecular interactions. By choosing the electrostatic interactions as the strong ones and the van der Waals interactions as the weak ones, we estimated the parameters of our model from these data (which represent the number of strong and weak bonds present at any time of the simulation). Numerical computations support our choice of the electrostatic interactions as the strong bonds, and the significance of the value found for $\Delta p_{s w}$ in that protein.

\section{Acknowledgments}

We wish to thank A. Finazzi Agrò and P. Baldi for their valuable discussions on the argument. We would like to express particular thanks to the referee for useful comments and suggestions which have improved the presentation of the whole paper. This work was partially supported by a grant of CNR.

\section{References}

1. M. Abundo, L. Accardi and N. Rosato, A Markovian model for cooperative interactions in proteins, Math. Models Methods Appl. Sci. 5 (1995) 835-863.

2. M. Abundo, L. Accardi, A. Finazzi Agrò, G. Mei and N. Rosato, A stochastic model for the sigmoidal behaviour of cooperative biological systems, Biophys. Chem. 58 (1996) 313-323.

3. M. Abundo and L. Caramellino, Some remarks about a Markov chain modelling cooperative biological systems, Open Systems Inform. Dynamics 3 (1995) 325-343.

4. M. Abundo, P. Baldi and L. Caramellino, $A$ diffusion approximation which models hierarchic interactions in cooperative biological systems, preprint Volterra No. 221 (1995), to appear in Open Systems Inform. Dynamics (1998).

5. A. Caflisch and M. Karplus, Acid and thermal denaturation of Barnase investigated by molecular dynamics simulations, J. Mol. Biol. 252 (1995) 672-708.

6. V. Daggett and M. Levitt, Protein unfolding pathways explored through molecular dynamics simulations, J. Mol. Biol. 232 (1993) 600-619.

7. S. N. Ethier and T. G. Kurtz, Markov Processes. Characterization and Convergence (Wiley, 1986).

8. D. Koroliuk and V. S. Koroliuk, Diffusion approximation of Markov chains for cooperative interactions in proteins, preprint Volterra (1994).

9. D. W. Strook and S. R. S. Varadhan, Multidimensional Diffusion Processes (Springer-Verlag, 1979). 